QUARTERLY OF APPLIED MATHEMATICS

VOLUME LXV, NUMBER 1

MARCH 2007, PAGES 43-68

S $0033-569 \mathrm{X}(07) 01050-\mathrm{X}$

Article electronically published on February 6, 2007

\title{
NON-LINEAR AND NON-STATIONARY MODES OF THE LOWER BRANCH OF THE INCOMPRESSIBLE BOUNDARY LAYER FLOW DUE TO A ROTATING DISK
}

\author{
BY \\ M. TURKYILMAZOGLU \\ Department of Mathematics, Hacettepe University, 06532-Beytepe, Ankara, Turkey
}

\begin{abstract}
In this paper a theoretical study is undertaken to investigate the structure of the lower branch neutral stability modes of three-dimensional small disturbances imposed on the incompressible Von Karman's boundary layer flow due to a rotating disk. Particular attention is given to the short-wavelength non-linear non-stationary crossflow vortex modes at sufficiently high Reynolds numbers with reasonably small scaled frequencies. Following closely the asymptotic frameworks introduced in [Proc. Roy. Soc. London Ser. A 406 (1986), 93-106] and [Proc. Roy. Soc. London Ser. A 413 (1987), 497-513] for the stationary linear and non-linear modes, it is revealed here that the nonstationary modes with sufficiently long time scale can also be described by an asymptotic expansion procedure based on the triple-deck theory. Making use of this approach, which takes into account the non-linear and non-parallel effects, the asymptotic structure of the non-stationary modes is shown to be adjusted by a balance between viscous and Coriolis forces, and resulted from the fact of vanishing shear stress at the disk surface. As a consequence of the matching of the solutions in adjacent regions it is found that in the linear case the wavenumber and the orientation of the lower branch modes are governed by an eigenrelation, which is akin to the one obtained previously in [Proc. Roy. Soc. London Ser. A 406 (1986), 93-106] for the stationary modes. The asymptotic theory shows that the non-parallelism has a destabilizing effect. A Landau-type equation for the modulated vortex amplitude with coefficients that are often difficult to get from finite Reynolds number computations has also been obtained from a weakly non-linear analysis in the limit of infinitely large Reynolds numbers. The non-linearity has also been found to be destabilizing for both positive and negative frequency waves, though finite amplitude growth of a disturbance having positive frequency close to the neutral location is more effective.
\end{abstract}

Received January 27, 2006.

2000 Mathematics Subject Classification. Primary 76U05; Secondary 76E09, 34K25.

Key words and phrases. Rotating-disk flow, stationary/non-stationary waves, triple-deck theory. Current address: Department of Mathematics, Hacettepe University, 06532-Beytepe, Ankara, Turkey.

E-mail address: turkyilm@hotmail.com

(C)2007 Brown University

Reverts to public domain 28 years from publication 
1. Introduction. The problem of understanding the phenomenon of transition from laminar flow to turbulence in fluid flows of practical importance has been the focus of past and recent studies in fluid dynamics, in particular with regard to the development of controlled laminar flow airfoils. It is generally recognized that various two- and threedimensional instability mechanisms, such as Görtler vortices, Tollmien-Schlichting waves and cross-flow vortices, render it difficult to maintain laminar flow on the surface of aircrafts and aerospace vehicles. The cross-flow instability is the most dangerous for the three-dimensional boundary layer flow on a swept wing or on the rotating disk, and thus, it is largely responsible for the breakdown of the laminar flow and transition to turbulence. The existence of such an instability is mainly owing to the inflectional character of the steady mean flow profiles. The flow due to a rotating disk also exhibits crossflow instability, since inflection points are also present in its basic velocity profiles. For this reason, in addition to the advantage of having an exact solution of the governing Navier-Stokes equations for the basic laminar Karman flow, the stability investigations of rotating-disk boundary layer flow have been the subject of many studies both experimental and theoretical.

The ongoing research on the flow due to a rotating disk indicates that the underlying cross-flow vortex instability may appear in two distinct forms, absolute and convective. The presence of the former was identified first in [3], and later extensively investigated in [4, 5] and [6, 7, 8, 9. In addition to this, the work of [10] pointed out the secondary absolute instability of the naturally selected primary non-linear cross-flow vortices. It can be inferred from these studies that the radially growing absolutely unstable disturbances may constitute a route to transition in rotating-disk flow, even though the wall compliance through surface coating may prevent the occurrence of absolute instability; see for instance [11.

When investigating a linear or non-linear development of an instability mode, it would be useful to distinguish between purely spatial analysis as implemented in this paper from the temporal or mixed spatio-temporal considerations. Thus, we here deal with the near-neutral stability of spatially developing disturbances in the limit of large Reynolds numbers, which may manifest themselves in the form of an inviscid upper branch (as first described by [12]) or of a viscous lower branch. The fundamental properties of these modes were investigated both experimentally and theoretically by many researchers, among them [6, 12, 13, 14, 15, 16, 17, 18, 19, 20. As suggested by these investigations, inviscid instability is characterized by the form of a stationary pattern due to the superposition of modes of zero-frequency spiral vortices, which align themselves at an inclination angle of approximately $13^{\circ}$ between the axis of vortices and the radius vector. Calculations predict the critical Reynolds number of about 300 for the inviscid instability mode. Moreover, theoretical works also indicate the existence of viscous instability which occurs with a much lower critical transition Reynolds number, readily depending on the frequency, than the inviscid stationary mode. This instability, which was also observed experimentally by [19, manifests itself as a wave pattern of spiral vortices inclined at a higher angle of about $20^{\circ}$ to the radius vector with a lower wavenumber than that corresponding to the inviscid instability of [12]. 
All of the aforementioned theoretical works have comprehensively used parallel-flow approximation methods, together with the replacement of the radius of the disk by the Reynolds number at some stage during the linearization of the governing equations, in order that the resulting system may be reduced to a more analyzable and compact form, describing the evolution of the small perturbations. Although such an approximation can give adequate results for sufficiently large Reynolds numbers, at finite Reynolds numbers it cannot be justified. Bearing this in mind, a more rigorous approach is needed, which not only accounts for the non-linearity and non-parallelism of the basic flow, but also relies on a large Reynolds number assumption for the formal asymptotic solutions of the full non-linear governing equations. Such a rational and self-consistent method is the asymptotic triple-deck theory; see for the description and its applications [21, 22], [23] and 24], which was first used within the context of predicting the stability features of the Blasius boundary layer flow in [25], 26] and [27]. As these papers demonstrate, this theory can allow for a more acceptable treatment of non-linearity, non-parallelism, streamline curvature and Coriolis effects belonging to the flow structure, and it enables the influence of the potential flow outside the boundary layer to be incorporated fully inside it. This strategy was successfully adapted to the linear instability exploration of rotating-disk flow in [1, in which both upper branch and lower branch stationary neutral modes and their asymptotic structures were obtained within the framework of asymptotic expansion at large Reynolds numbers.

Making use of the asymptotic triple-deck theory, the linear and non-linear evolution of the upper branch modes of the rotating-disk boundary layer flow, as far as the orientation of the non-stationary waves is concerned, were examined in [28] and [29]. These modes are the ones naturally observed in the experiments of [12, 17] and [30, 31. However, as first detected in the experiment of [19, there exist lower branch modes corresponding to a lower Reynolds number. In [2] an investigation was performed (making use of the theory developed in 1]) of the non-linear stability properties on the lower branch instability modes, and it was noticed that these modes can be observed in real flows provided that the external perturbations are strong enough; otherwise, the inviscid modes of [12] would be dominant. In addition to this, the recent experiment of 32 clearly pointed out that under the natural transition process non-stationary disturbances are first amplified, even though at later stages the transition is dominated by the stationary waves. If, on the other hand, the system is forced with a roughness situated on the rotating disk, the transition was observed in 33 to take place through the travelling perturbations. This sets forth the significance of the non-stationary waves, and therefore our main aim here is to extend the work of [2] to incorporate the effects of non-linearity on such disturbances near the position of neutral stability, and as a result to determine whether the stationary or non-stationary waves augment each other to cause the exponential amplification and transition. Actually, a smaller finite amplitude disturbance with a positive frequency has been found to be sufficient to give rise to an exponential growth of the solution.

Numerical calculations of [15] and asymptotic work of [1] have justified that the lower branch short-wavelength stationary viscous mode corresponds to zero wall shear stress of the effective cross-flow velocity profiles. In addition to this, parallel-flow approximation results of [6] and [20] clearly demonstrate that not only the zero-frequency waves 
but also the non-stationary waves with small frequencies approach the zero wall shear stress at high Reynolds numbers. Motivated in particular by this observation, in the current research another objective is to calculate the non-stationary lower branch neutral modes having sufficiently long time scales and as a consequence determine how the lower branch modes of [20] will be modified by the non-parallel effects. As shown in [1, the lower branch modes are governed by a triple-deck structure, for which the upper and main-deck flow description is essentially the same as that found in 26] for the Blasius boundary layer flow. Basically the same expansions are also supposed to be valid for the non-stationary neutral waves considered here. We found here that the lower-deck expansions yield an eigenrelation involving parabolic cylinder functions, connecting the flow parameters. Moreover, the non-parallelism accounted for in the present work is found to be destabilizing, though the wavenumber and wave angle computed from this asymptotic description compare fairly well with the numerical solution of full stability equations based on the parallel-flow assumption. The final objective of the current work is to identify the occurrence of the bifurcation at the lower branch of the stability curve for a range of positive frequencies, first spotted in the numerical study [20]. Our investigation is an attempt to study this behavior analytically within a self-consistent non-parallel-flow approximation. Using the approach outlined here, further analysis can also be implemented to investigate the growing waves having small frequencies.

The rest of the paper is organized in the following fashion. In $\S 2$ the non-linear partial differential equations governing the stability of the incompressible boundary layer flow over a rotating disk are given together with the basic Von Karman's steady flow. $§ 3$ is concerned with the construction of a set of asymptotic expansions and the solutions of the corresponding asymptotic equations of the flow behavior in each asymptotic region within the concept of triple-deck theory. Both linear and non-linear approximations are considered in $\S 3$. The linear results are first compared with the numerical ones of [6] and [20] in $\S 4.1$, followed by the determination of an amplitude function from a weakly non-linear analysis in $\S 4.2$. Finally our conclusions are drawn in $\S 5$.

2. Formulation of the problem. We are concerned with the motion of a threedimensional viscous incompressible flow occupying the $z \geq 0$ region and adjacent to a disk rotating with a constant angular velocity $\Omega_{a}$. The flow has kinematic viscosity $\nu$, and the cylindrical polar coordinates $(r, \theta, z)$ are introduced, in which the lengths $r$ and $z$ have been made dimensionless with respect to a reference length scale $l$ that can be taken to be the local radius of the disk. The characterizing parameter, i.e. the Reynolds number of the flow, is defined as $R=\Omega_{a} l^{2} / \nu$. Since the reference frame attached to the disk rotates with it, the basic flow is formulated by the Von Karman solution with the velocity components $(u, v, w)$ and pressure $p$ given by

$$
(u, v, w, p)=l \Omega_{a}\left(r \bar{u}(Y), r \bar{v}(Y), R^{-1 / 2} \bar{w}(Y), R^{-1} \bar{p}(Y)\right),
$$

which satisfy the following differential equations:

$$
\begin{array}{r}
\bar{u}^{2}-(\bar{v}+1)^{2}+\bar{u}^{\prime} \bar{w}-\bar{u}^{\prime \prime}=0, \\
2 \bar{u}(\bar{v}+1)+\bar{v}^{\prime} \bar{w}-\bar{v}^{\prime \prime}=0 \\
2 \bar{u}+\bar{w}^{\prime}=0
\end{array}
$$


which should be solved subject to the boundary conditions

$$
\begin{aligned}
& \bar{u}=0, \quad \bar{v}=0, \quad \bar{w}=0, \quad \text { at } Y=0, \\
& \bar{u}=0, \quad \bar{v}=-1, \quad \bar{w}=w_{c}, \quad \text { as } Y \rightarrow \infty,
\end{aligned}
$$

where the prime denotes differentiation with respect to $Y=R^{1 / 2} z$, and $w_{c}$ is a constant to be found as a result of the solution of equations (2.1)-(2.2). Moreover, the steady pressure component can also be obtained from the normal momentum equation, but it is of no relevance to the subsequent analysis.

The dynamic flow field is next decomposed into a mean flow plus perturbations. Thus the mean flow determined from (2.1) is perturbed with small, unsteady, three-dimensional disturbances of the form

$$
l \Omega_{a}(\tilde{U}(r, \theta, z, t), \tilde{V}(r, \theta, z, t), \tilde{W}(r, \theta, z, t), \tilde{P}(r, \theta, z, t)) .
$$

After substituting these into the Navier-Stokes equations we find that the evolution of the perturbations is governed by the following non-linear equations:

$$
\begin{aligned}
& \left\{L_{1}+\bar{u}\right\} \tilde{U}-2(\bar{v}+1) \tilde{V}+r \frac{d \bar{u}}{d z} \tilde{W}-\frac{1}{r} \tilde{V}^{2}+L_{2} \tilde{U} \\
& =-\frac{\partial \tilde{P}}{\partial r}+\frac{1}{R}\left\{\nabla \tilde{U}-\frac{2}{r^{2}} \frac{\partial \tilde{V}}{\partial \theta}-\frac{\tilde{U}}{r^{2}}\right\}, \\
& \left\{L_{1}+\bar{u}\right\} \tilde{V}+2(\bar{v}+1) \tilde{U}+r \frac{d \bar{v}}{d z} \tilde{W}+\frac{1}{r} \tilde{U} \tilde{V}+L_{2} \tilde{V} \\
& =-\frac{1}{r} \frac{\partial \tilde{P}}{\partial \theta}+\frac{1}{R}\left\{\nabla \tilde{V}+\frac{2}{r^{2}} \frac{\partial \tilde{U}}{\partial \theta}-\frac{\tilde{V}}{r^{2}}\right\}, \\
& \left\{L_{1}+R^{-1 / 2} \frac{d \bar{w}}{d z}\right\} \tilde{W}+L_{2} \tilde{W}=-\frac{\partial \tilde{P}}{\partial z}+\frac{1}{R}\{\nabla \tilde{W}\}, \\
& \frac{\partial \tilde{U}}{\partial r}+\frac{\tilde{U}}{r}+\frac{1}{r} \frac{\partial V}{\partial \theta}+\frac{\partial \tilde{W}}{\partial z}=0 .
\end{aligned}
$$

Here, the linear operators $L_{1}, \nabla$ and non-linear operator $L_{2}$ are defined by $L_{1}=\frac{\partial}{\partial t}+$ $r \bar{u} \frac{\partial}{\partial r}+\bar{v} \frac{\partial}{\partial \theta}+R^{-1 / 2} \bar{w} \frac{\partial}{\partial z}, \nabla=\frac{\partial^{2}}{\partial r^{2}}+\frac{1}{r} \frac{\partial}{\partial r}+\frac{1}{r^{2}} \frac{\partial^{2}}{\partial \theta^{2}}+\frac{\partial^{2}}{\partial z^{2}}$, and $L_{2}=\tilde{U} \frac{\partial}{\partial r}+\frac{\tilde{V}}{r} \frac{\partial}{\partial \theta}+\tilde{W} \frac{\partial}{\partial z}$, respectively. Equations (2.3) are subject to the condition of zero-slip on the wall, and also the condition of attenuation of the perturbations far away from the wall above the disk is enforced. The imposition of such a constraint is sufficient, though not necessary, see [34, to keep in line with the results of much of the available works in the area so that comparisons can be adequately implemented. [34, on the other hand, showed recently the existence of disturbances which are physically conceivable as well as growing away from the wall of the disk, which we shall not deal with here.

3. Asymptotic regions. Our aim in the current investigation is to determine asymptotic solutions of the non-linear perturbation equations (2.3) in the limit of large Reynolds number, in each of the asymptotic regimes as depicted in Figure 1. It is convenient here to define a small parameter $\varepsilon$ by $\varepsilon=R^{-1 / 16}$. This quantity arises from the consideration of the concept of triple deck. The reason for this small parameter is that in parallel-flow approximation (see [1]), the typical wavenumber of the neutrally stable modes on the lower branch increases proportionally to $R^{1 / 4}$ as $R \rightarrow \infty$. For the viscous lower branch modes then, the thickness of the viscous wall layer (region III) is $O\left(R^{-1 / 2} \varepsilon\right)=O\left(\varepsilon^{9}\right)$, and the thickness of the main layer (region II) is $O\left(R^{-1 / 2}\right)=O\left(\varepsilon^{8}\right)$. Moreover, because of the fact that the wavenumbers in the $r$ and $\theta$ directions are of order of magnitude $R^{1 / 4}$, see for instance 1 and 12, the thickness of the upper deck (region I) will be $O\left(R^{-1 / 4}\right)=O\left(\varepsilon^{4}\right)$. Based on the above triple-deck scalings, the linear 
stationary problem was discussed in [1] and the non-linear stationary problem in [2]. The asymptotic regions are shown in Figure 1.

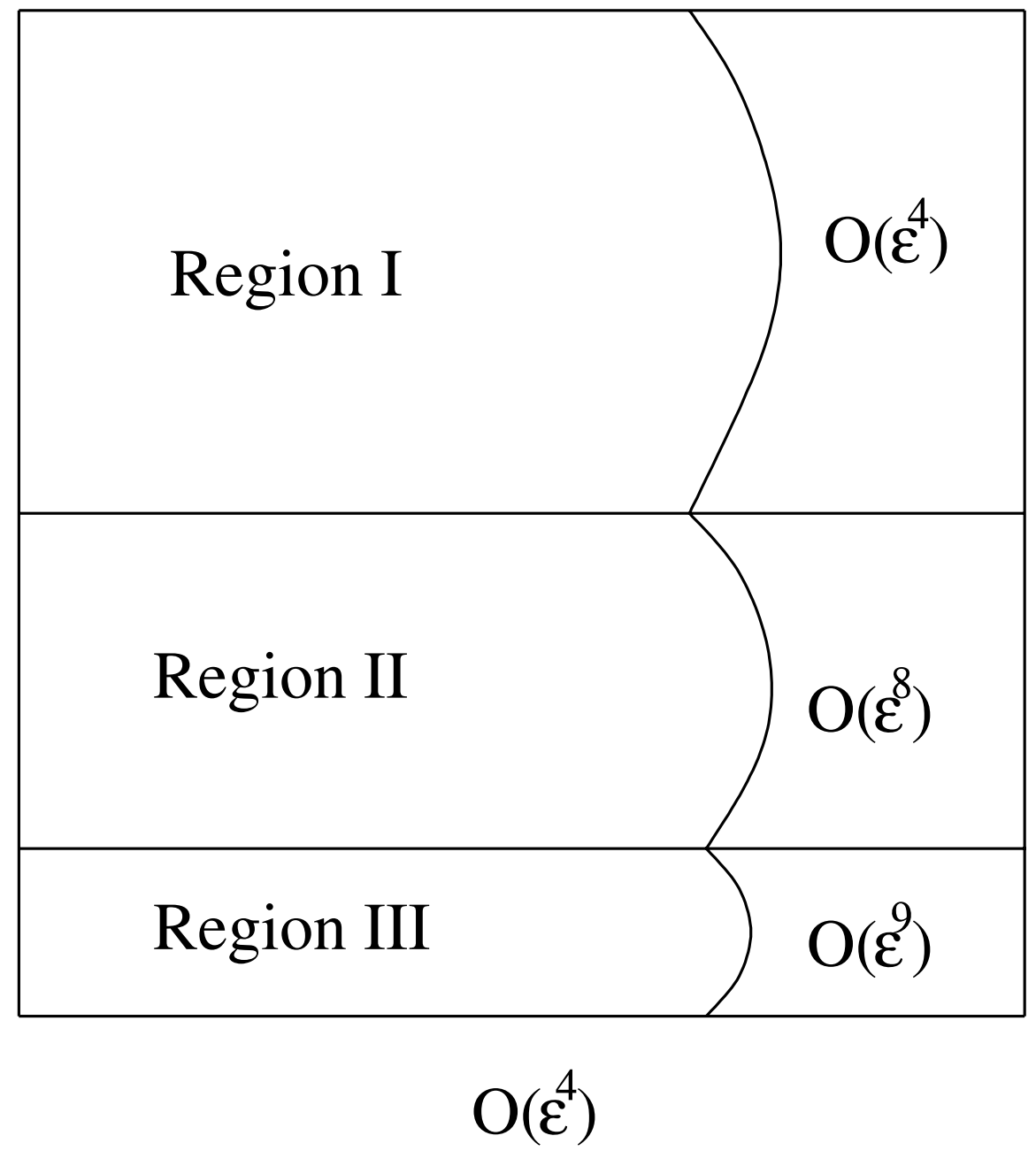

FIG. 1. Asymptotic regions of the triple deck and thicknesses of each layer are shown in terms of a small parameter $\varepsilon=R^{-1 / 16}$; region I corresponds to the upper deck, region II to the main deck and region III to the lower deck.

It was shown in 12 that the effective velocity profile with wavenumbers $\alpha$ and $\beta$ in the $r$ and $\theta$ directions, respectively, is given by $\alpha r \bar{u}+\beta \bar{v}$. Lower branch disturbances having the triple-deck structure are necessarily time-dependent if the effective wall shear $\left(\alpha r \bar{u}^{\prime}+\beta \bar{v}^{\prime}\right)(0)$ does not vanish. However, as the work of [1] points out, the zero-frequency lower branch neutral modes have vanishing shear. It was further shown theoretically in [6] and 20] that the orientation of high-frequency lower branch neutral modes approaches the direction of maximum mean wall shear, whereas the wavenumbers of the neutral 
modes with small frequency approach the neutral curve asymptotically for the zerofrequency mode. Therefore, we are here concerned only with the non-stationary modes having small frequencies, which enable us to follow the asymptotic expansion procedure set up in [1, and hence we look for solutions proportional to

$$
E=e^{\frac{i}{\varepsilon^{4}}\left[\int^{r} \alpha(r) d r+\beta \theta-\varepsilon^{2} \omega t\right]},
$$

where the wavenumbers $(\alpha, \beta)$ and frequency $\omega$ expand for convenience in terms of the small parameter $\varepsilon$ as

$$
\begin{aligned}
& \alpha=\alpha_{0}+\varepsilon^{2} \alpha_{1}+\varepsilon^{3} \alpha_{2}+\cdots, \\
& \beta=\beta_{0}+\varepsilon^{2} \beta_{1}+\varepsilon^{3} \beta_{2}+\cdots, \\
& \omega=\varepsilon \omega_{0}+\varepsilon^{2} \omega_{1}+\varepsilon^{3} \omega_{2} \cdots .
\end{aligned}
$$

Here we search for the local wavenumber and frequency components which contribute to the neutrally stable flow initially at a radial location $r$, corrected later by a finite amplitude non-linear solution. Moreover, it should also be remembered here that unlike the linearized equations, the expansions of the disturbances for the non-linear solutions will involve harmonics generated by the convective and non-linear terms in the perturbation equations (2.3). Furthermore, we should address here that the periodicity in the azimuthal direction is ensured by considering a single azimuthal harmonic of the form (3.1) with the physical mode number $\varepsilon^{-4} \beta$ asymptotically large in the limit of large Reynolds numbers. The fact that $\varepsilon^{-4} \beta$ is an integer implies a discretization either through $\varepsilon$ or $\beta_{j}, j=0,1, \cdots$ as given in (3.2). However, because of the fact that we define an effective wavenumber $k$ (that depends on $\gamma_{0}$ given later by equation (4.1) in $\S 4.1$ ) in place of $\alpha$ and $\beta$, it will be unimportant to assume whether the values of $\beta$ are discrete or not. In addition to this, though the asymptotic expansion for $\varepsilon^{-4} \beta$ will terminate at a finite place, it won't influence our analysis below since only the consideration of lower-order terms in $\beta$ will suffice for our purposes (see the Landau-type equation (4.18) in $\S 4.2$ ).

Following closely the study of [2], next the wavenumbers and frequency as given in (3.1) and the disturbances will be substituted in the perturbed Navier-Stokes equations (2.3), and solutions to the expansions of the flow quantities will be sought separately in each asymptotic region as shown in Figure 1.

3.1. Region I. We begin with the analysis of the external potential flow region, namely, the upper deck, where the cylindrical polar coordinates $r$ and $\theta$ are of the order of unity in the limit of large Reynolds number, and the motion is inviscid and irrotational.

In the potential flow region the order one normal coordinate is introduced as

$$
Z=\varepsilon^{-4} z=O(1),
$$

and the basic velocity components are simply

$$
\bar{u}=0, \quad \bar{v}=-1, \quad \bar{w}=w_{c} .
$$

Introducing further a small amplitude $\delta$ (which is later related to $\varepsilon$ in $\S 4.2$ ), perturbations proportional to powers of $\delta$ driven by the non-linear terms expand as

$$
\begin{gathered}
\tilde{U}=\varepsilon^{3}\left(U_{u 0}+\varepsilon U_{u 1}+\cdots\right) \delta E+\left(U_{u 20}+\varepsilon U_{u 21}+\cdots\right) \delta^{2} E^{2} \\
+\varepsilon^{-3}\left(U_{u 30}+\varepsilon U_{u 31}+\cdots\right) \delta^{3} E^{3}+\varepsilon^{-3}\left(U_{u 10}+\varepsilon U_{u 11}\right. \\
+\cdots) \delta^{3} E+O\left(\delta^{4}\right)+\text { c.c. }
\end{gathered}
$$


together with a similar expansion for $\tilde{V}$, while $\tilde{W}$ expands as

$$
\begin{gathered}
\tilde{W}=\varepsilon^{3}\left(W_{u 0}+\varepsilon W_{u 1}+\cdots\right) \delta E+\delta^{2}\left[\left(W_{u 20}+\varepsilon W_{u 21}+\cdots\right) E^{2}\right. \\
\left.+\varepsilon^{5}\left(W_{u m 0}+\varepsilon W_{u m 1}+\cdots\right)\right]+\varepsilon^{-3}\left(W_{u 30}+\varepsilon W_{u 31}+\cdots\right) \delta^{3} E^{3} \\
+\varepsilon^{-3}\left(W_{u 10}+\varepsilon W_{u 11}+\cdots\right) \delta^{3} E+O\left(\delta^{4}\right)+c . c .
\end{gathered}
$$

and $\tilde{P}$ expands as

$$
\begin{gathered}
\tilde{P}=\varepsilon^{3}\left(P_{u 0}+\varepsilon P_{u 1}+\cdots\right) \delta E+\delta^{2}\left[\left(P_{u 20}+\varepsilon P_{u 21}+\cdots\right) E^{2}\right. \\
\left.+\varepsilon^{6}\left(P_{u m 0}+\varepsilon P_{u m 1}+\cdots\right)\right]+\varepsilon^{-3}\left(P_{u 30}+\varepsilon P_{u 31}+\cdots\right) \delta^{3} E^{3} \\
+\varepsilon^{-3}\left(P_{u 10}+\varepsilon P_{u 11}+\cdots\right) \delta^{3} E+O\left(\delta^{4}\right)+c . c .
\end{gathered}
$$

where c.c. denotes complex conjugate terms, $E$ is defined by (3.1) together with $\alpha, \beta$ and $\omega$ as given by (3.2), and $U_{u 0}, V_{u 0}, W_{u 0}, P_{u 0}$, etc. are functions of $r$ and $Z$. Notice also the main-flow correction terms $W_{u m 0}$ and $P_{u m 0}$, entering into the upper-deck flow region. Substituting these asymptotic expansions into the governing non-linear disturbed equations (2.3) and equating coefficients of like powers of $\varepsilon$ from the terms proportional to $\delta E$ and solving the resulting equations, one can find that solutions to the leading-order equations which decay to zero at the far field are given by

$$
\begin{aligned}
& P_{u 0}=C_{1} e^{-\gamma_{0} Z}, U_{u 0}=\frac{\alpha_{0}}{\beta_{0}} C_{1} e^{-\gamma_{0} Z}, \\
& V_{u 0}=\frac{C_{1}}{r} e^{-\gamma_{0} Z}, W_{u 0}=i \frac{\gamma_{0}}{\beta_{0}} C_{1} e^{-\gamma_{0} Z},
\end{aligned}
$$

in which the zero-order effective wavenumber $\gamma_{0}$ having positive real part for the nonneutral waves is defined as $\gamma_{0}^{2}=\alpha_{0}^{2}+\frac{\beta_{0}^{2}}{r^{2}}$ and $C_{1}$ is an unknown amplitude function of $r$. Moreover, solutions to the next-order equations are the same as those given in (3.6) except that $C_{1}$ is substituted by $D_{1}$, which is also a function of $C_{1}$. Furthermore, the form of the solutions of the disturbance equations resulting from the terms proportional to $\delta^{2} E^{2}, \delta^{3} E^{3}$ and $\delta^{3} E$ in equations (3.3)-(3.5) is similar to (3.6), apart from the integration constants $C_{1}$ and $D_{1}$ being replaced in each case by $C_{i}$ and $D_{i}, i=2,3,4$, which are functions of $C_{1}(r)$, too. Finally, the leading-order mean flow correction terms, due to the terms proportional to $\delta^{2}$ are found to be $W_{u m 0}=C_{2}$ and $P_{u m 0}=C_{2}$.

It should finally be remarked here that the solutions obtained up to this order are the same as the solutions of the stationary problem explored in 2. However, the nonzero frequency feature will come in later within the higher-order terms in (3.3)-(3.5), in particular through a term containing $\omega_{0}$.

3.2. Region II. In this region, occupying the bulk of the boundary layer, the asymptotic analysis of the Navier-Stokes equations is based on the limit process

$$
Y=\varepsilon^{-8} z=O(1), \text { as } R e \rightarrow \infty .
$$

Expanding the asymptotic solution (3.6) (and also the higher-order solutions) in the upper deck in terms of the scaling $Y$ of region II suggests that the asymptotic expansion of $\tilde{U}$ in the main deck should be in the form

$$
\begin{aligned}
& \tilde{U}=\varepsilon^{-1}\left(U_{m 0}+\varepsilon U_{m 1}+\cdots\right) \delta E+\delta^{2}\left[\varepsilon^{-4}\left(U_{m 20}+\varepsilon U_{m 21}+\cdots\right) E^{2}\right. \\
& \left.+\varepsilon^{-3}\left(u_{m 0}+\varepsilon u_{m 1}+\cdots\right)\right]+\varepsilon^{-7}\left(U_{m 30}+\varepsilon U_{m 31}+\cdots\right) \delta^{3} E^{3} \\
& +\varepsilon^{-7}\left(U_{m 10}+\varepsilon U_{m 11}+\cdots\right) \delta^{3} E+O\left(\delta^{4}\right)+c . c .
\end{aligned}
$$


together with a similar expression for $\tilde{V}$, whereas $\tilde{W}$ expands as

$$
\begin{aligned}
& \tilde{W}=\varepsilon^{3}\left(W_{m 0}+\varepsilon W_{m 1}+\cdots\right) \delta E+\delta^{2}\left[\left(W_{m 20}+\varepsilon W_{m 21}+\cdots\right) E^{2}\right. \\
& \left.+\varepsilon^{5}\left(w_{m 0}+\varepsilon w_{m 1}+\cdots\right)\right]+\varepsilon^{-3}\left(W_{m 30}+\varepsilon W_{m 31}+\cdots\right) \delta^{3} E^{3} \\
& +\varepsilon^{-3}\left(W_{m 10}+\varepsilon W_{m 11}+\cdots\right) \delta^{3} E+O\left(\delta^{4}\right)+c . c .
\end{aligned}
$$

and $\tilde{P}$ expands as

$$
\begin{aligned}
& \tilde{P}=\varepsilon^{3}\left(P_{m 0}+\varepsilon P_{m 1}+\cdots\right) \delta E+\delta^{2}\left[\left(P_{m 20}+\varepsilon P_{m 21}+\cdots\right) E^{2}\right. \\
& \left.+\varepsilon^{6}\left(p_{m 0}+\varepsilon p_{m 1}+\cdots\right)\right]+\varepsilon^{-3}\left(P_{m 30}+\varepsilon P_{m 31}+\cdots\right) \delta^{3} E^{3} \\
& +\varepsilon^{-3}\left(P_{m 10}+\varepsilon P_{m 11}+\cdots\right) \delta^{3} E+O\left(\delta^{4}\right)+c . c .
\end{aligned}
$$

The variables appearing in equations (3.7)-3.9) are all functions of $r$ and $Y$. Making again the usual substitution into the non-linear perturbed Navier-Stokes equations (2.3) and after matching with the upper-deck results of (3.3) -(3.6) will generate, for the quantities proportional to the $\delta E$ terms, the following solutions to the leading-order terms in the asymptotic expansions (3.7)-(3.9):

$$
\begin{aligned}
& P_{m 0}=C_{1}, U_{m 0}=r \frac{\gamma_{0}}{\beta_{0}^{2}} C_{1} \bar{u}^{\prime}, \\
& V_{m 0}=r \frac{\gamma_{0}}{\beta_{0}^{2}} C_{1} \bar{v}^{\prime}, W_{m 0}=-i \frac{\gamma_{0}}{\beta_{0}^{2}} C_{1}\left(\alpha_{0} r \bar{u}+\beta_{0} \bar{v}\right) .
\end{aligned}
$$

The next-order terms of $\delta E$ in the asymptotic expansions (3.7)-(3.9) have similar solutions to (3.10). Equations (3.10) also indicate that $\tilde{W}$ satisfies the no-slip condition on the wall, even though $U_{m 0}$ and $V_{m 0}$ do not. However, the lower branch neutral modes that we consider here possess the feature of having zero shear stress on the wall at the first order (as first shown in [1]). Thus, imposition of such a constraint on the modes yields $\alpha_{0} r \bar{u}^{\prime}(0)+\beta_{0} \bar{v}^{\prime}(0)=0$, which in turn determines a relation for the leading-order eigenvalues as $\frac{\alpha_{0} r}{\beta_{0}}=1.207$. Moreover, as suggested by the numerical results of [6], the above relation will hold true for small frequency waves as well; though there occurs a shift in $W_{m 3}$ across the boundary layer through the leading-order frequency term $\omega_{0}$, the associated part is given by

$$
W_{m 3}=\frac{i \omega_{0} C_{1}}{\beta_{0}^{2}} \omega_{0},
$$

which will be eventually taken care of through the lower-deck expansion below.

Additionally, solutions to the terms proportional to $\delta^{2} E^{2}, \delta^{3} E^{3}$ and $\delta^{3} E$ in the expansions (3.7)-(3.9) have the same forms as (3.10) with $\alpha_{0}, \beta_{0}$ and $\omega_{0}$, etc., replaced by $2 \alpha_{0}$ etc. and $3 \alpha_{0}$ etc. respectively, with the amplitudes to match with the upper deck solutions. Finally, the leading-order mean flow corrections coming from the terms proportional to $\delta^{2}$ in (3.7)-(3.9) are

$$
u_{m 0}=\frac{r \gamma_{0} C_{2}}{2 \beta_{0}^{2}} \bar{u}^{\prime}, \quad v_{m 0}=\frac{r \gamma_{0} C_{2}}{2 \beta_{0}^{2}} \bar{v}^{\prime}, \quad w_{m 0}=-\frac{\gamma_{0} C_{2}}{\beta_{0}^{2}} \bar{u}
$$


3.3. Region III. This region, corresponding to the lower deck in Figure 1, allows us to satisfy the no-slip condition on the disk surface. As mentioned earlier, in the limit of large Reynolds number the thickness of this region is such that

$$
\zeta=\varepsilon^{-9} z=O(1)
$$

The expansion of the basic flow quantities in terms of the lower-deck scaling are

$$
\begin{aligned}
& \bar{u}=\varepsilon \bar{u}_{0} \zeta+\varepsilon^{2} \bar{u}_{1} \zeta^{2}+\cdots, \\
& \bar{v}=\varepsilon \bar{v}_{0} \zeta+\varepsilon^{2} \bar{v}_{1} \zeta^{2}+\cdots, \\
& \bar{w}=-\varepsilon^{2} \bar{u}_{0} \zeta^{2}-\frac{2}{3} \varepsilon^{3} \bar{u}_{1} \zeta^{3}+\cdots
\end{aligned}
$$

Substituting (3.10) - (3.13) into (3.7)-(3.9) and expressing the results in terms of $\zeta$ suggests that in the lower-deck function, $\tilde{U}$ should be written as

$$
\begin{aligned}
& \tilde{U}=\varepsilon^{-1}\left[r A_{1}\left(\bar{u}_{0}+2 \varepsilon \bar{u}_{1} \zeta+\cdots\right)+\left(U_{-1}+\varepsilon U_{0}+\cdots\right)\right] \delta E \\
& +\delta^{2} \varepsilon^{-4}\left\{\left[r \frac{A_{2}}{2}\left(\bar{u}_{0}+2 \varepsilon \bar{u}_{1} \zeta+\cdots\right)+\left(U_{20}+\varepsilon U_{21}+\cdots\right)\right] E^{2}\right. \\
& \left.+\varepsilon\left(U_{m 0}+\varepsilon U_{m 1}+\cdots\right)\right\}+\varepsilon^{-7}\left[r \frac{A_{3}}{3}\left(\bar{u}_{0}+2 \varepsilon \bar{u}_{1} \zeta+\cdots\right)\right. \\
& \left.+\left(U_{30}+\varepsilon U_{31}+\cdots\right)\right] \delta^{3} E^{3}+\varepsilon^{-7}\left[r A_{4}\left(\bar{u}_{0}+2 \varepsilon \bar{u}_{1} \zeta+\cdots\right)\right. \\
& \left.+\left(U_{10}+\varepsilon U_{11}+\cdots\right)\right] \delta^{3} E+O\left(\delta^{4}\right)+\text { c.c. }
\end{aligned}
$$

Asymptotic expansion for $\tilde{V}$ has the same form, while $\tilde{W}$ expands as

$$
\begin{aligned}
& \tilde{W}=\varepsilon^{5}\left\{-i A_{1}\left[\left(r \alpha_{0} \bar{u}_{1}+\beta_{0} \bar{v}_{1}\right) \zeta^{2}+\varepsilon\left(r \alpha_{0} \bar{u}_{2}+\beta_{0} \bar{v}_{2}\right) \zeta^{3}+\cdots\right]\right. \\
& \left.+\left(\varepsilon W_{0}+\cdots\right)\right\} \delta E+\delta^{2} \varepsilon^{2}\left\{\left[-i A_{2}\left[\left(r \alpha_{0} \bar{u}_{1}+\beta_{0} \bar{v}_{1}\right) \zeta^{2}\right.\right.\right. \\
& \left.\left.+\varepsilon\left(r \alpha_{0} \bar{u}_{2}+\beta_{0} \bar{v}_{2}\right) \zeta^{3}+\cdots\right]+\left(\varepsilon W_{20}+\cdots\right)\right] E^{2} \\
& \left.+\varepsilon^{4}\left(W_{m 0}+\varepsilon W_{m 1}+\cdots\right)\right\}+\left\{-i A_{3} \varepsilon^{-1}\left[\left(r \alpha_{0} \bar{u}_{1}+\beta_{0} \bar{v}_{1}\right) \zeta^{2}\right.\right. \\
& \left.\left.+\varepsilon\left(r \alpha_{0} \bar{u}_{2}+\beta_{0} \bar{v}_{2}\right) \zeta^{3}+\cdots\right]+\left(W_{30}+\cdots\right)\right\} \delta^{3} E^{3} \\
& +\left\{-i A_{4} \varepsilon^{-1}\left[\left(r \alpha_{0} \bar{u}_{1}+\beta_{0} \bar{v}_{1}\right) \zeta^{2}+\varepsilon\left(r \alpha_{0} \bar{u}_{2}+\beta_{0} \bar{v}_{2}\right) \zeta^{3}+\cdots\right]\right. \\
& \left.+\left(W_{10}+\cdots\right)\right\} \delta^{3} E+O\left(\delta^{4}\right)+c . c .
\end{aligned}
$$

and $\tilde{P}$ expands as

$$
\begin{aligned}
& \tilde{P}=\varepsilon^{3}\left(P_{0}+\varepsilon P_{1}+\cdots\right) \delta E+\delta^{2}\left[\left(P_{20}+\varepsilon P_{21}+\cdots\right) E^{2}\right. \\
& \left.+\varepsilon^{10}\left(P_{m 0}+\varepsilon P_{m 1}+\cdots\right)\right]+\varepsilon^{-3}\left(P_{30}+\varepsilon P_{31}+\cdots\right) \delta^{3} E^{3} \\
& +\varepsilon^{-3}\left(P_{10}+\varepsilon P_{11}+\cdots\right) \delta^{3} E+O\left(\delta^{4}\right)+c . c .
\end{aligned}
$$

where $A_{i}=\frac{\gamma_{0} C_{i}}{\beta_{0}^{2}}, i=1,2,3,4$. The coefficients in the expansions (3.14)-(3.16) are functions of $r$ and $\zeta$.

After substituting (3.14)-(3.16) into the non-linear disturbance equations (2.3), and also equating the same order- $\varepsilon$ terms from the resulting equations, we obtain the following successive equations from the continuity and radial momentum equations, respectively, 
for the terms proportional to $\delta E$ :

$$
\begin{aligned}
& \quad i\left(r \alpha_{0} U_{-1}+\beta_{0} V_{-1}\right)=0 \\
& \quad i\left(r \alpha_{0} U_{0}+\beta_{0} V_{0}\right)=0 \\
& \quad i\left(r \alpha_{0} U_{1}+\beta_{0} V_{1}\right)+r W_{0}^{\prime}=-i r A_{1}\left[r \alpha_{1} \bar{u}_{0}+\beta_{1} \bar{v}_{0}\right] \\
& \quad-i\left(r \alpha_{1} U_{-1}+\beta_{1} V_{-1}\right) \\
& \quad i\left(r \alpha_{0} U_{2}+\beta_{0} V_{2}\right)+r W_{1}^{\prime}=-2 i r A_{1}\left[r \alpha_{1} \bar{u}_{1}+\beta_{1} \bar{v}_{1}\right] \zeta \\
& \quad-i r A_{1}\left(r \alpha_{2} \bar{u}_{0}+\beta_{2} \bar{v}_{0}\right)-i\left(r \alpha_{1} U_{0}+\beta_{1} V_{0}\right)-i\left(r \alpha_{2} U_{-1}+\beta_{2} V_{-1}\right), \\
& U_{-1}^{\prime \prime}-i\left(r \alpha_{0} \bar{u}_{1}+\beta_{0} \bar{v}_{1}\right) \zeta^{2} U_{-1}=0 \\
& U_{0}^{\prime \prime}-i\left(r \alpha_{0} \bar{u}_{1}+\beta_{0} \bar{v}_{1}\right) \zeta^{2} U_{0}=r \bar{u}_{0} W_{0} \\
& +i\left\{\left(r \alpha_{1} \bar{u}_{0}+\beta_{1} \bar{v}_{0}\right) \zeta-\omega_{0}\right\}\left\{A_{1} r \bar{u}_{0}+U_{-1}\right\}+i\left(r \alpha_{0} \bar{u}_{2}+\beta_{0} \bar{v}_{2}\right) \zeta^{3} U_{-1}, \\
& U_{1}^{\prime \prime}-i\left(r \alpha_{0} \bar{u}_{1}+\beta_{0} \bar{v}_{1}\right) \zeta^{2} U_{1}=2 r \bar{u}_{1} \zeta W_{0}+i \alpha_{0} P_{0}-2 V_{-1} \\
& +i\left(r \alpha_{0} \bar{u}_{2}+\beta_{0} \bar{v}_{2}\right) \zeta^{3} U_{0}+i\left\{\left(r \alpha_{1} \bar{u}_{0}+\beta_{1} \bar{v}_{0}\right) \zeta-\omega_{0}\right\}\left\{2 A_{1} r \bar{u}_{1} \zeta+U_{0}\right\} \\
& +i\left(r \alpha_{1} \bar{u}_{1}+\beta_{1} \bar{v}_{1}\right) \zeta^{2}\left[A_{1} r \bar{u}_{0}+U_{-1}\right]+i\left(r \alpha_{0} \bar{u}_{3}+\beta_{0} \bar{v}_{3}\right) \zeta^{4} U_{-1} \\
& +i\left(r \alpha_{2} \bar{u}_{0}+\beta_{2} \bar{v}_{0}\right) \zeta\left[A_{1} r \bar{u}_{0}+U_{-1}\right] \\
& U_{2}^{\prime \prime}-i\left(r \alpha_{0} \bar{u}_{1}+\beta_{0} \bar{v}_{1}\right) \zeta^{2} U_{2}=2 r \bar{u}_{1} \zeta W_{1}+3 r \bar{u}_{2} \zeta^{2} W_{0}+i \alpha_{0} P_{1} \\
& -2 \bar{v}_{0} \zeta V_{-1}-2 V_{0}+i\left\{\left(r \alpha_{1} \bar{u}_{0}+\beta_{1} \bar{v}_{0}\right) \zeta-\omega_{0}\right\}\left\{3 A_{1} r \bar{u}_{2} \zeta^{2}+U_{1}\right\} \\
& +i\left(r \alpha_{1} \bar{u}_{1}+\beta_{1} \bar{v}_{1}\right) \zeta^{2}\left[2 A_{1} r \bar{u}_{1} \zeta+U_{0}\right]+\bar{u}_{0} \zeta U_{-1} \\
& +i\left(r \alpha_{1} \bar{u}_{2}+\beta_{1} \bar{v}_{2}\right) \zeta^{3}\left[A_{1} r \bar{u}_{0}+U_{-1}\right]+i\left(r \alpha_{0} \bar{u}_{2}+\beta_{0} \bar{v}_{2}\right) \zeta^{3} U_{1} \\
& +i\left(r \alpha_{0} \bar{u}_{3}+\beta_{0} \bar{v}_{3}\right) \zeta^{4} U_{0}+i\left(r \alpha_{0} \bar{u}_{4}+\beta_{0} \bar{v}_{4}\right) \zeta^{5} U_{-1} \\
& +i\left(r \alpha_{2} \bar{u}_{0}+\beta_{2} \bar{v}_{0}\right) \zeta\left[2 A_{1} r \bar{u}_{1} \zeta+U_{0}\right] \\
& +i\left(r \alpha_{2} \bar{u}_{1}+\beta_{2} \bar{v}_{1}\right) \zeta^{2}\left[A_{1} r \bar{u}_{0}+U_{-1}\right]
\end{aligned}
$$

with similar equations for $\tilde{V}$ from the azimuthal momentum equation, but for $\tilde{P}$ from the normal momentum equation we get

$$
P_{0}^{\prime}=0, \quad P_{1}^{\prime}=0
$$

It is worth noting here that the term $-2 V_{-1}$ appearing on the right-hand side of the third equation in (3.18) (and also $2 U_{-1}$ in the corresponding azimuthal momentum equation) is due to the Coriolis effects, showing that the structure of the neutral modes of small frequency waves depends upon both viscous and Coriolis effects. The above system of equations is considered together with the following boundary conditions, some of which stem directly from matching with the main-deck solutions in $§ 3.2$ :

$$
\begin{aligned}
& U_{-1}(0)=-A_{1} r \bar{u}_{0}, \quad U_{-1}(\infty)=0, \quad V_{-1}(0)=-A_{1} r \bar{v}_{0}, \quad V_{-1}(\infty)=0, \\
& U_{0}(0)=0, \quad U_{0}(\infty)=B_{1} r \bar{u}_{0}, \quad V_{0}(0)=0, \quad V_{0}(\infty)=B_{1} r \bar{v}_{0}, \\
& W_{0}(0)=0, \quad W_{0}^{\prime}(0)=0, \quad W_{0}(\infty)=-i A_{1}\left\{\left(r \alpha_{1} \bar{u}_{0}+\beta_{1} \bar{v}_{0}\right) \zeta-\omega_{0}\right\}, \\
& W_{1}(0)=0, \quad W_{1}^{\prime}(0)=0, \quad W_{1}(\infty)=-i A_{1}\left(\alpha_{1} r \bar{u}_{1}+\beta_{1} \bar{v}_{1}\right) \zeta^{2},
\end{aligned}
$$

where $B_{1}$ is defined in the same way as $A_{1}$ but $C_{1}$ is replaced by $D_{1}$. The wall derivative conditions in (3.20) are obtained using the third and fourth equations in (3.17). In addition to this, the matching of the pressure in regions II and III through equations (3.10) and (3.19) produces $P_{0}=C_{1}$ and $P_{1}=D_{1}$.

So far we have obtained governing equations for the small linearized disturbances $(\delta E$ terms only) related to each corresponding region of the triple deck; see Figure 1. After a little lengthy work, it is straightforward to show that terms proportional to $\delta^{2} E^{2}, \delta^{3} E^{3}$ 
and $\delta^{3} E$ produce equations similar to (3.17)-(3.20) with slight changes arising from the non-linear and convective effects in equation (2.3). Now, using equations (3.17)-(3.20) we shall deduce an eigenequation relating $\gamma_{0}$ to the wavenumber $\beta_{0}$ and frequency $\omega_{0}$.

We see that the first of equations (3.18) together with the corresponding boundary conditions may be solved for $U_{-1}$; the solution has the form

$$
U_{-1}=-A r \bar{u}_{0} \frac{U_{c}\left(0, \sqrt{2} s_{1}\right)}{U_{c}(0,0)},
$$

with $s_{1}=\Delta^{1 / 4} \zeta$ and $\Delta=i\left(r \alpha_{0} \bar{u}_{1}+\beta_{0} \bar{v}_{1}\right)$. Here $U_{c}$ denotes the parabolic cylinder function; see, for instance, [35. A similar solution holds for $V_{-1}$.

In order to obtain the solutions for $U_{0}$ and $V_{0}$ we multiply the third equation in (3.18) and the equation for $V_{0}$ by $i r \alpha_{0}$ and $i \beta_{0}$ respectively. We then add them together and, making use of (3.17), arrive at a conclusion that

$$
\begin{aligned}
& W_{0}^{\prime \prime \prime}-i\left(r \alpha_{0} \bar{u}_{1}+\beta_{0} \bar{v}_{1}\right) \zeta^{2} W_{0}^{\prime}+2 i\left(r \alpha_{0} \bar{u}_{1}+\beta_{0} \bar{v}_{1}\right) \zeta W_{0} \\
& =\gamma_{0}^{2} C_{1}-2 i \frac{\beta_{0}}{r}\left[1+\frac{\bar{v}_{0}^{2}}{\bar{u}_{0}^{2}}\right] U_{-1}+A_{1} \zeta\left(r \alpha_{0} \bar{u}_{1}+\beta_{0} \bar{v}_{1}\right)\left\{\left(r \alpha_{1} \bar{u}_{0}+\beta_{1} \bar{v}_{0}\right) \zeta-2 \omega_{0}\right\} .
\end{aligned}
$$

The solution of this equation satisfying the condition of matching with the solution in region II may be written in the form

$$
\begin{aligned}
& W_{0}=-i A_{1}\left(r \alpha_{1} \bar{u}_{0}+\beta_{1} \bar{v}_{0}\right) \Delta^{-1 / 4} s_{1}+i A_{1} \omega_{0} F_{1}\left(s_{1}\right) \\
& +\left\{\gamma_{0}^{2} C_{1} F_{2}\left(s_{1}\right)+2 i \beta_{0} A_{1}\left[1+\frac{\bar{v}_{0}^{2}}{\bar{u}_{0}^{2}}\right] \frac{\bar{u}_{0}}{U_{c}(0,0)} F_{3}\left(s_{1}\right)\right\} \Delta^{-3 / 4}+k_{1} \zeta^{2},
\end{aligned}
$$

where $k_{1}$ is an arbitrary constant.

The functions $F_{1}, F_{2}$ and $F_{3}$ used in (3.22) satisfy the following Weber-like differential equation (see [35]):

$$
\begin{aligned}
& F_{1}^{\prime \prime \prime}-s_{1}^{2} F_{1}^{\prime}+2 s_{1} F_{1}=2 s_{1}, \quad F_{1}(0)=0, F_{1}(\infty)=1, F_{1}^{\prime}(\infty)=0, \\
& F_{2}^{\prime \prime \prime}-s_{1}^{2} F_{2}^{\prime}+2 s_{1} F_{2}=1, \quad F_{2}(0)=0, F_{2}(\infty)=0, F_{2}^{\prime}(\infty)=0, \\
& F_{3}^{\prime \prime \prime}-s_{1}^{2} F_{3}^{\prime}+2 s_{1} F_{3}=U_{c}\left(0, \sqrt{2} s_{1}\right), \\
& F_{3}(0)=0, F_{3}(\infty)=0, F_{3}^{\prime}(\infty)=0 .
\end{aligned}
$$

The boundary conditions for $F_{1}, F_{2}$ and $F_{3}$ have been formulated using (3.20).

It can be shown through the direct numerical solution of (3.23) that (alternatively the contour integration suggested in [1] may be used)

$$
\begin{aligned}
& I_{1}=F_{1}^{\prime}(0)=1.3520 \\
& I_{2}=F_{2}^{\prime}(0)=\frac{1}{2 U_{c}(0,0)} \int_{0}^{\infty} \theta U_{c}(0, \theta) d \theta=0.5990, \\
& I_{3}=2 \frac{F_{3}^{\prime}(0)}{U_{c}(0,0)}=\frac{1}{U_{c}^{2}(0,0)} \int_{0}^{\infty} \theta U_{c}^{2}(0, \theta) d \theta=0.4570 .
\end{aligned}
$$

Finally, imposing the condition $W_{0}^{\prime}(0)=0$ leads to the sought eigenrelation

$$
i A_{1} \Delta^{1 / 2}\left(r \alpha_{1} \bar{u}_{0}+\beta_{1} \bar{v}_{0}\right)=i A_{1} \omega_{0} \Delta^{3 / 4} I_{1}+\gamma_{0}^{2} C_{1} I_{2}+i \beta_{0} A_{1}\left[1+\frac{\bar{v}_{0}^{2}}{\bar{u}_{0}^{2}}\right] \bar{u}_{0} I_{3} .
$$

It is easy to notice that this relation differs from the one obtained in [1] and [2] through the presence of the frequency $\omega_{0}$. Denoting $U^{0}=\left[1+\frac{\bar{v}_{0}^{2}}{\bar{u}_{0}^{2}}\right]^{1 / 2}, \overline{u v}_{0}=\left|\bar{u}_{0} \bar{v}_{0}\right|^{1 / 2}$ 
and $\Phi_{0}=\left[\frac{\alpha_{1}}{\beta_{0}}-\frac{\beta_{1} \alpha_{0}}{\beta_{0}^{2}}\right]$, and separating the real and imaginary parts in (3.25), we find

$$
\begin{aligned}
& r \frac{\overline{u v} 0}{2} \beta_{0}^{-1 / 2} \Phi_{0}=\gamma_{0} I_{2}+\sin (3 \pi / 8)\left(2 \bar{u}_{0}\right)^{-3 / 4}\left|\bar{v}_{0}\right|^{3 / 4} I_{1} \beta_{0}^{-5 / 4} \omega_{0}, \\
& r \frac{\overline{u v} 0}{2} \beta_{0}^{-1 / 2} \Phi_{0}=\frac{U^{0^{2}}}{\beta_{0}} \bar{u}_{0} I_{3}+\cos (3 \pi / 8)\left(2 \bar{u}_{0}\right)^{-3 / 4}\left|\bar{v}_{0}\right|^{3 / 4} I_{1} \beta_{0}^{-5 / 4} \omega_{0} .
\end{aligned}
$$

Since the left-hand sides of these equations are equal, so should be the right-hand sides, which gives rise to the following eigenrelation:

$$
a \Omega+b \gamma^{1 / 4}-\gamma^{9 / 4}=0,
$$

describing the neutral stability of the flow at its current radial position, say, $r=\bar{r}$. Here $a_{1}=\sin \left(\frac{3 \pi}{8}\right) \frac{I_{1}}{I_{2}}\left(\frac{-\bar{v}_{0}}{2 \bar{u}_{0}}\right)^{3 / 4} U^{0^{5 / 4}}, a_{2}=a_{1} \cot \left(\frac{3 \pi}{8}\right), a=a_{2}-a_{1}, b=\bar{u}_{0} U^{0^{3}} I_{3} / I_{2}, \gamma_{0}=r^{-1 / 2} \gamma$ and $\omega_{0}=r^{1 / 8} \Omega$.

By equating coefficients of like powers of $\varepsilon$ from the resulting disturbance equations after substituting terms proportional to $\delta^{2} E^{2}$ given in (3.14)-(3.16), and a likewise manipulation of the obtained equations as implemented for (3.17)-(3.21) yields the leadingorder normal velocity term

$$
\begin{aligned}
& W_{20}=-i A_{2}\left(r \alpha_{1} \bar{u}_{0}+\beta_{1} \bar{v}_{0}\right) \Delta^{-1 / 4} s_{2}+i A_{2} \omega_{0} F_{1}\left(s_{2}\right) \\
& +\left\{4 \gamma_{0}^{2} C_{2} F_{2}\left(s_{2}\right)+2 i \beta_{0} A_{2}\left[1+\frac{\bar{v}_{0}^{2}}{\bar{u}_{0}^{2}}\right] \frac{\bar{u}_{0}}{U_{c}(0,0)} F_{3}\left(s_{2}\right)\right\}(2 \Delta)^{-3 / 4}+O\left(\zeta^{2}\right),
\end{aligned}
$$

where $s_{2}=(2 \Delta)^{1 / 4} \zeta$. From the continuity equation concerning $\delta^{2} E^{2}$ terms we get equations similar to (3.17) and, hence, the zero normal derivative on the wall produces the relation

$$
\begin{aligned}
& C_{2}\left\{4 \gamma_{0}^{2} I_{2}+i \frac{\gamma_{0}}{\beta_{0}} U^{0^{2}} \bar{u}_{0} I_{3}-i \frac{\gamma_{0}}{\beta_{0}^{2}}(2 \Delta)^{1 / 2}\left(r \alpha_{1} \bar{u}_{0}+\beta_{1} \bar{v}_{0}\right)\right. \\
& \left.+i \frac{\gamma_{0}}{\beta_{0}^{2}} \omega_{0} I_{1}(2 \Delta)^{3 / 4}\right\}=C_{1}^{2} \frac{\gamma_{0}^{0}}{\beta_{0}^{4}}(2 \Delta)^{3 / 2},
\end{aligned}
$$

which associates the amplitude $C_{2}$ of the first harmonic to $C_{1}$.

A similar argument as above generates the amplitude $C_{3}$ of the second harmonic, which appears to be related to $C_{1}$ through the relation

$$
\begin{aligned}
& C_{3}\left\{9 \gamma_{0}^{2} I_{2}+i \frac{\gamma_{0}}{\beta_{0}} U^{0^{2}} \bar{u}_{0} I_{3}-2 i \frac{\gamma_{0}}{\beta_{0}^{2}}(3 \Delta)^{1 / 2}\left(r \alpha_{1} \bar{u}_{0}+\beta_{1} \bar{v}_{0}\right)\right. \\
& \left.+\frac{3 i}{2} \frac{\gamma_{0}}{\beta_{0}^{2}} \omega_{0} I_{1}(3 \Delta)^{3 / 4}\right\}=C_{2} C_{1}^{*} \frac{\gamma_{0}^{2}}{\beta_{0}^{4}}(3 \Delta)^{3 / 2},
\end{aligned}
$$

in which the term on the right-hand side is due to the non-linearity of the governing equations and ${ }^{*}$ denotes complex conjugate.

Next we proceed to the terms proportional to $\delta^{3} E$, which give rise to equations such as (3.17)-(3.20). As implemented above the relation linking the amplitude $C_{4}$ of the third harmonic to $C_{1}$ is found to be

$$
\begin{aligned}
& C_{4}\left\{-i \frac{\gamma_{0}}{\beta_{0}^{2}}\left(r \alpha_{1} \bar{u}_{0}+\beta_{1} \bar{v}_{0}\right)+\Delta^{-1 / 2}\left[\gamma_{0}^{2} I_{2}+i \frac{\gamma_{0}}{\beta_{0}} U^{0^{2}} \bar{u}_{0} I_{3}\right]\right. \\
& \left.+i \frac{\gamma_{0}}{\beta_{0}^{2}} \omega_{0} I_{1} \Delta^{1 / 4}\right\}=C_{2} C_{1}^{*} \frac{\gamma_{0}^{2}}{\beta_{0}^{4}} \Delta .
\end{aligned}
$$

Finally, together with the anticipation of zero-slip on the wall as well as the match with the main deck, the leading-order mean-flow correction terms proportional to $\delta^{2}$ in (3.14) - (3.16) are obtained as

$$
U_{m 0}=r A_{2} \bar{u}_{1} \zeta, \quad V_{m 0}=r A_{2} \bar{v}_{1} \zeta, \quad W_{m 0}=-A_{2} \bar{u}_{1} \zeta^{2} .
$$




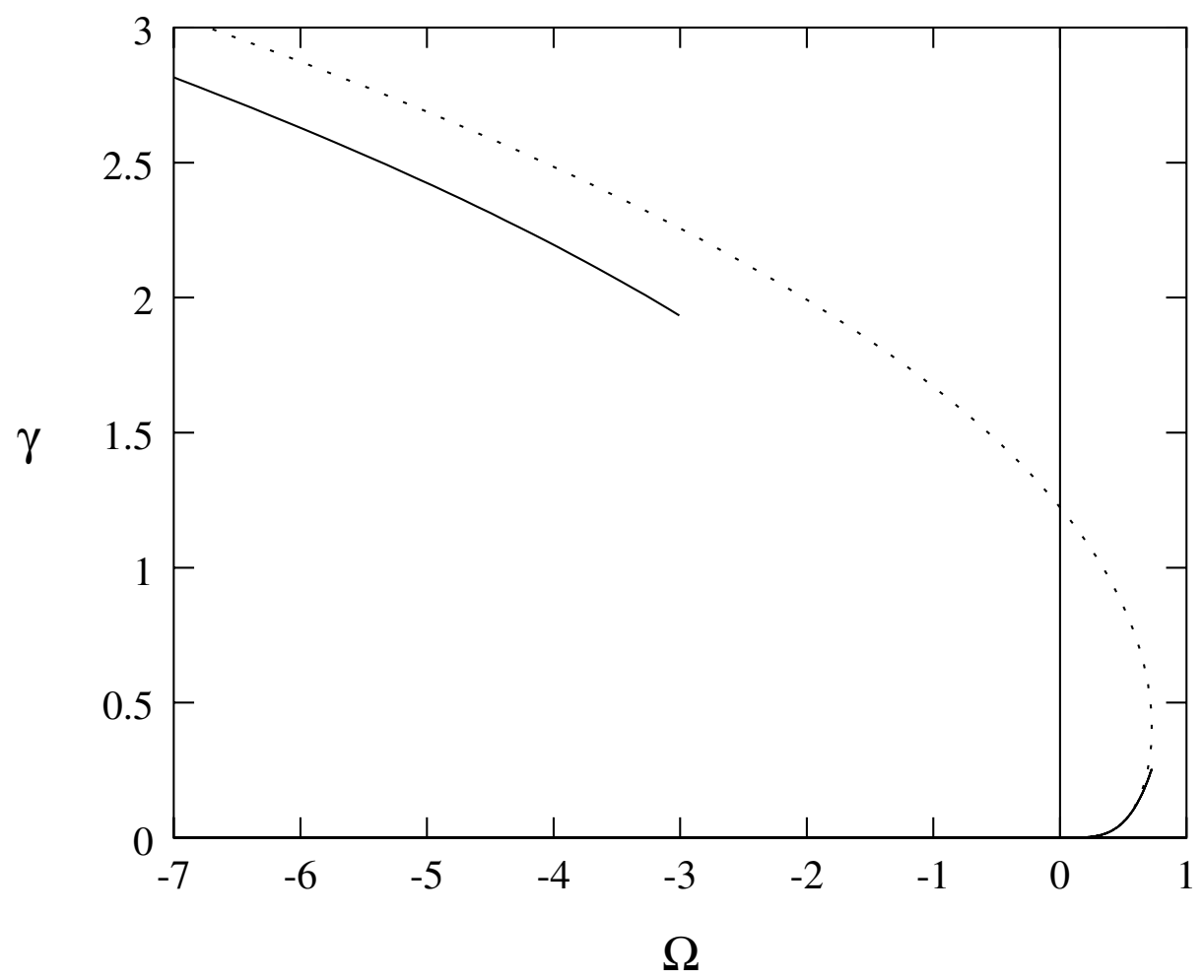

FIG. 2. The solution of eigenrelation (3.27) is presented as the leading-order wavenumber versus the frequency by the dotted curve. Also the asymptotes for large and small $\gamma$ are shown by the black curves.

\section{Results and discussion.}

4.1. Linear results ( $\delta E$ terms). The leading-order wavenumber is governed by relation (3.27), which is associated with the non-zero frequency neutral disturbances. It is straightforward to check that the solution of (3.27) in the limit of the stationary neutral mode of 1 results in $\gamma=1.22$. Figure 2 demonstrates the calculated roots (and also the asymptotes for large and small $\gamma$, which are indicated by the unbroken curves) that are real and positive, and so connected to the physical problem as initially imposed. The solution to equation (3.27) is in fact characterized by a parameter $\Omega_{c}$. Therefore, beyond the value of $\Omega_{c}$ (which can be calculated from (3.27) as $\Omega_{c}=-\frac{8}{a}\left(\frac{b}{9}\right)^{9 / 8}$ ) it is apparent from Figure 2 that no non-stationary modes exist. Thus, $\Omega_{c}$ constitutes a cut-off value for the positive frequency waves. This should be no surprise since within the present asymptotic theory sufficiently small frequencies with vanishing shear stress on the wall are assumed.

Another intriguing feature visible from Figure 2 is that for $0<\Omega<\Omega_{c}$, two real positive roots exist corresponding to two lower branch neutral modes of the non-stationary waves for asymptotically large Reynolds numbers. The existence of such a double mode was also observed in the numerical calculations of 20]. It was found in 20] (see Figure 7 
of [20]) that for some positive range of frequency, the relevant neutral curves approach the neutral curve of zero-frequency, after having turned around as the Reynolds number increases. The present theoretical work thus predicts such a behavior bringing with itself, in addition, an imposition of a threshold value for the frequency.

The critical layer analysis of [28] for the evaluation of non-stationary modes seems not capable of capturing the modes encountered here. This might be due to the reason that the critical layer analysis does not yield negative frequencies and only points out the stationary mode of [1]. However, within the current asymptotic approach, since the shearing stress at the wall is presumed to diminish at the leading-order, the nonstationary neutral waves behaving qualitatively in a manner similar to the stationary one are well captured in the large Reynolds number limit. In addition to this, the wave angle, that is, the angle that wave disturbances make with the radius of the disc lies between $0^{\circ}$ and $90^{\circ}$, a much wider range than that found in [28].

It is also clear from Figure 2 that for $\Omega<0$ the mode matching to the stationary mode of [1] has an effective wavenumber $\gamma$ that increases monotonically as the frequency decreases. This behavior is also consistent with the numerical solution, as presented later, of the full linearized stability equations obtained by a parallel-flow assumption.

Having solved for the leading-order wavenumber expression, the correction term to the inclination angle, $\Phi_{0}$, can be formulated by utilizing (3.26) as

$$
\Phi_{0}=\left(\gamma^{3 / 2}+a_{1} \gamma^{-3 / 4} \Omega\right) b_{1} I_{2} r^{-5 / 4}=\phi r^{-5 / 4}
$$

with $b_{1}=2\left|\bar{u}_{0} \bar{v}_{0} U^{0}\right|^{-1 / 2}$. The scaled wave angle correction term $\phi$ versus the frequency is shown in Figure 3, which is also consistent with the stationary mode of [1, leading to a value of 2.3 for $\Omega=0$. It is seen that positive frequency waves make a smaller angle of inclination with the radius vector than the negative frequency waves.

However, since we have not yet found $\alpha_{1}$ and $\beta_{1}$ separately, the correction term to the wavenumber cannot be determined without further continuing the next-order terms. In this connection, using the findings so far, the total effective wavenumber can be expressed by

$$
k=\sqrt{\alpha^{2}+\frac{\beta^{2}}{r^{2}}}=\gamma_{0}\left[1+\left(\alpha_{0} \alpha_{1}+\frac{\beta_{0} \beta_{1}}{r^{2}}\right) \varepsilon^{2} / \gamma_{0}^{2}+\cdots\right]
$$

In a similar fashion, the inclination angle between the radius and cross-flow vortices, defined by $\Phi=\arctan \left(\frac{\beta}{\alpha r}\right)$, satisfies

$$
\begin{aligned}
& \cot \Phi=\tan \left(\frac{\pi}{2}-\Phi\right)=\frac{\alpha_{0} r}{\beta_{0}}+\left[\frac{\alpha_{1}}{\beta_{0}}-\frac{\alpha_{0} \beta_{1}}{\beta_{0}^{2}}\right] r \varepsilon^{2}+\cdots \\
& =1.207+\phi r^{-1 / 4} \varepsilon^{2}+\cdots
\end{aligned}
$$

In order to be able to compare the present asymptotic results (4.1)- 4.2) with the available numerical stability results, such as [6], the following adjustments need to be done first. Noting that $R_{\Delta}, k_{\Delta}$ and $\omega_{\Delta}$ denote the Reynolds number, wavenumber and frequency respectively, used in [6] and [15], and that $R_{\Delta}=r R^{1 / 2}$ (here $R_{\Delta}$ is the Reynolds number based on the boundary layer thickness and the local azimuthal velocity 


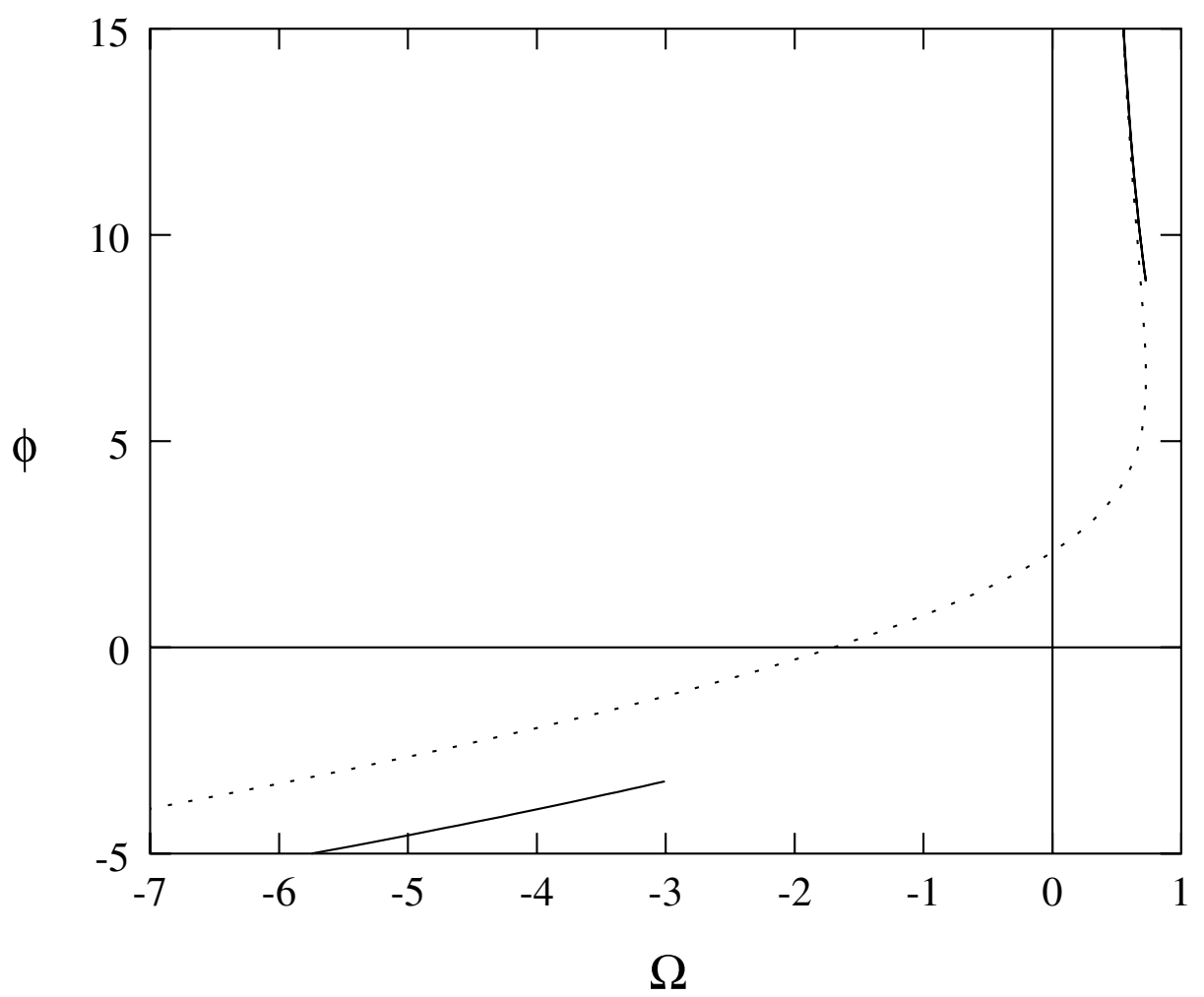

FIG. 3. The solution of eigenrelation (3.27) is presented as the correction angle versus the frequency by the dotted curve. Also the asymptotes for large and small $\gamma$ are shown by the black curves.

on the disk), the following relations are present:

$$
\begin{gathered}
k_{\Delta}=\varepsilon^{4} k r^{-1 / 2} \sim \gamma R_{\Delta}^{-1 / 2}, \\
\cot \Phi=\tan \left(\frac{\pi}{2}-\Phi\right) \sim 1.207+\phi R_{\Delta}^{-1 / 4}, \\
\omega_{\Delta} \sim \varepsilon^{-1} \frac{\omega_{0}}{R_{\Delta}^{1 / 8}}=\Omega .
\end{gathered}
$$

It is easy to ascertain from equations (4.3)- (4.5) that, by expressing the neutral values found in this investigation in terms of $R_{\Delta}$, the explicit dependence on the radial variable has been removed. It should also be remarked here that simply assigning $\Omega$ to zero in (4.5) will effectively produce the stationary modes, which were already treated in [1]. Another intriguing point which deserves attention is that, unlike the other boundary layer flows such as the Blasius flow (see [26]), here the stability quantities and as a result the structure of the lower branch modes arise from a balance between the viscous forces and Coriolis effects. Unlike this again, the eigenrelation determining the upper branch modes arises from a balance between various jumps across the critical layers and Stokes layer shift; see for instance 29. 


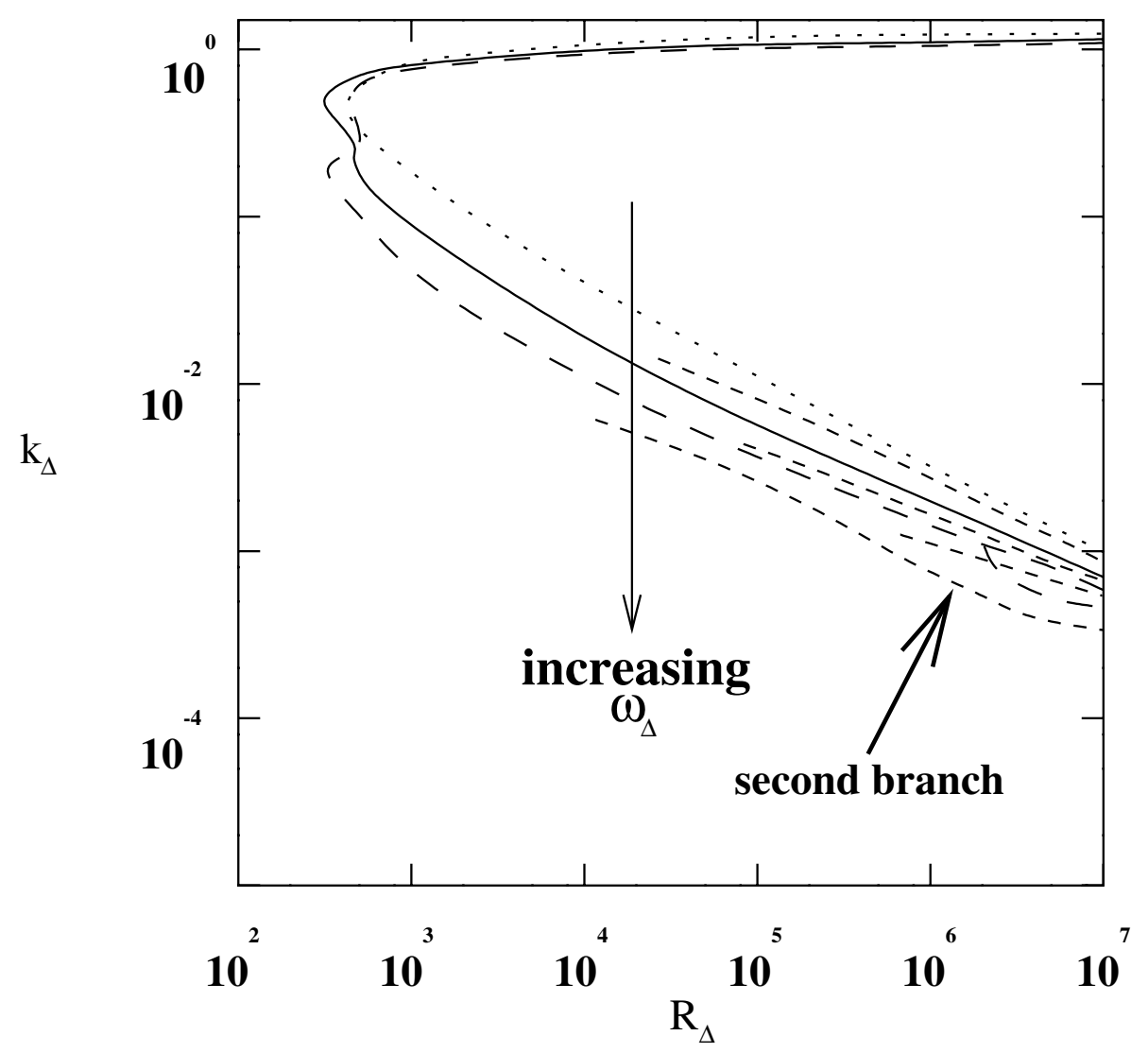

FIG. 4. Illustration of the variation of effective wavenumber against the Reynolds number for a variety of frequencies: (...) $\omega_{\Delta}=-2.5$, $(-) \omega_{\Delta}=0.0$, and (--) $\omega_{\Delta}=0.7$. The asymptotic structure of the non-stationary lower branch modes is also shown by broken lines (--). The second branch corresponding to $\omega_{\Delta}=0.7$ is also shown by the arrow.

In Figures 4 and 5, a comparison has been made between the computed numerical results of parallel-flow approximation (see [6]) and the asymptotic predictions as obtained from equations (4.3)-(4.5). It is seen for the limiting case of zero-frequency that the stationary viscous mode is perfectly recovered, showing the consistency of our asymptotic extension of the disturbance structure as set up in [1] to non-stationary small frequency modes. For small amplitude disturbances, the inviscid modes displayed as upper branch curves in Figure 4 are the dominant ones, whereas for larger amplitudes, the non-stationary short-wavelength modes depicted as lower branch curves might be more important, since then it might be possible that this class of modes may bifurcate subcritically as far as the non-linearity is concerned. The existence of such short-wavelength lower branch modes demonstrated in Figures 4 and 5 was first observed experimentally in [19], and recently in [32, 33. The second branch (indicated by the bold arrow) is most probably the one discovered in the experiment of [19]. 


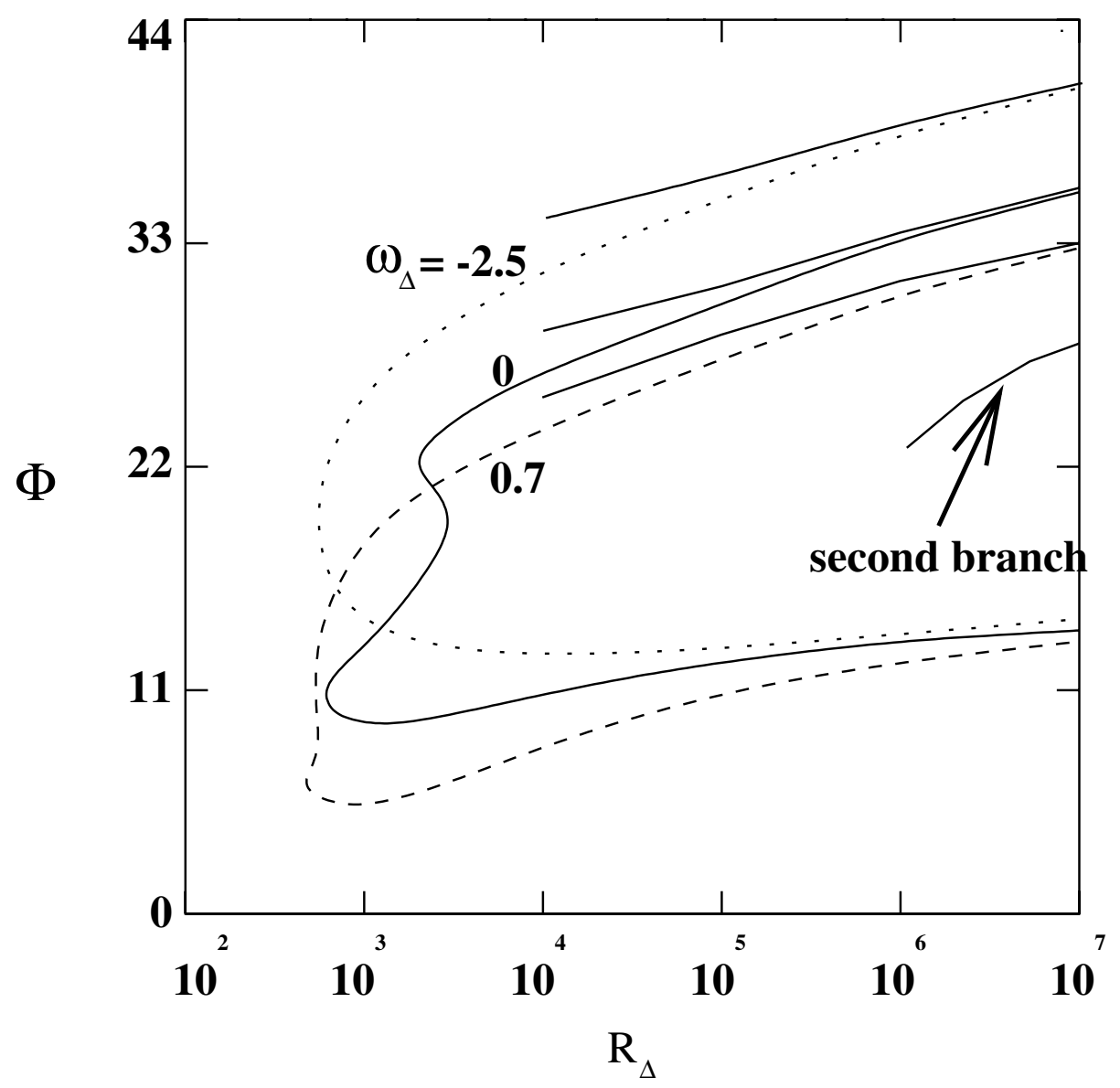

FIG. 5. Illustration of the variation of wave angle against the Reynolds number for a variety of frequencies: (..) $\omega_{\Delta}=-2.5,(-)$ $\omega_{\Delta}=0.0$ and (--) $\omega_{\Delta}=0.7$. The asymptotic structure of the nonstationary lower branch modes is also shown as unbroken lines for each $\omega_{\Delta}$ displayed. The second branch corresponding to $\omega_{\Delta}=0.7$ is also shown by the arrow.

To summarize this section, the contributions due to the boundary layer growth, that is non-parallelism, seem to be effectively destabilizing for the rotating-disk boundary layer flow taking into consideration the leading-order approximation. The dominant effect of the non-parallelism on the stability properties is appreciably felt for finite Reynolds numbers. For sufficiently large Reynolds numbers a fairly enhanced agreement occurs between the asymptotic and numerical findings. It would be interesting to further work out the contributions of non-parallelism which would arise from the consideration of higher-order terms in equations (4.1)-(4.2), within the asymptotic expansion technique pursued in this study.

4.2. Results of finite amplitude effects. From equation (3.31), which is satisfied at the position of neutral stability, say $r=\bar{r}$, we see that taking into account the relation 
(3.25), the left-hand side is zero when $r=\bar{r}$. Therefore, in order to determine a finite amplitude solution, we must move sufficiently away from the neutral radius to allow $C_{1}$ to be non-zero. Taking this into consideration, as in 2 we perturb the solution from the position of neutral stability by writing

$$
r=\bar{r}+\epsilon r_{1} .
$$

Within a similar inspection as implemented in [2, by choosing $\delta=\varepsilon^{7 / 2}$, a LandauGinzburg type equation is then formed to search for the effects of finite amplitude $C_{1}$; see also [36] and [37. To be concise, we pursue the argument given in [2] and omit the details, except that $\alpha_{2}$ in (3.17)-(3.19) is effectively replaced by

$$
\alpha_{2}=-i \frac{\partial}{\partial r_{1}} \text {. }
$$

Accounting for the above arguments, now, from (3.14) the lower-deck disturbance velocity $\tilde{U}$ will expand as

$$
\begin{aligned}
& \tilde{U}=\varepsilon^{7 / 2}\left\{\left[\varepsilon^{-1} \bar{r} A_{1}\left(\bar{u}_{0}+2 \varepsilon \bar{u}_{1} \zeta+\cdots\right)+\varepsilon^{-1}\left(\bar{U}_{-1}+\varepsilon \bar{U}_{0}+\cdots\right)\right] E\right. \\
& +\varepsilon^{-1 / 2}\left[\bar{r} \frac{A_{2}}{2}\left(\bar{u}_{0}+2 \varepsilon \bar{u}_{1} \zeta+\cdots\right)+\left(\bar{U}_{20}+\varepsilon \bar{U}_{21}+\cdots\right)\right] E^{2} \\
& +\varepsilon^{1 / 2}\left(\bar{U}_{m 0}+\varepsilon \bar{U}_{m 1}+\cdots\right)+\left[\bar{r} \frac{A_{3}}{3}\left(\bar{u}_{0}+2 \varepsilon \bar{u}_{1} \zeta+\cdots\right)\right. \\
& \left.\left.+\left(\bar{U}_{30}+\varepsilon \bar{U}_{31}+\cdots\right)\right] E^{3}\right\}+O\left(\varepsilon^{4}\right)+c . c .
\end{aligned}
$$

together with a similar expression for $\tilde{V}$, while $\tilde{W}$ and $\tilde{P}$ expand as

$$
\begin{aligned}
& \tilde{W}=\varepsilon^{7 / 2}\left\{\left\{-\varepsilon^{5} i A_{1}\left[\left(\alpha_{0} r \bar{u}_{1}+\beta_{0} \bar{v}_{1}\right) \zeta^{2}+\cdots\right]+\varepsilon^{6}\left(\bar{W}_{0}+\varepsilon \bar{W}_{1}+\cdots\right)\right\} E\right. \\
& +\varepsilon^{1 / 2}\left[-\varepsilon^{5} i A_{2}\left[\left(\alpha_{0} r \bar{u}_{1}+\beta_{0} \bar{v}_{1}\right) \zeta^{2}+\cdots\right]+\varepsilon^{6}\left(\bar{W}_{20}+\varepsilon \bar{W}_{21}+\cdots\right)\right] E^{2} \\
& +\varepsilon^{19 / 2}\left(\bar{W}_{m 0}+\varepsilon \bar{W}_{m 1}+\cdots\right)+\left\{-\varepsilon^{6} i A_{3}\left[\left(\alpha_{0} r \bar{u}_{1}+\beta_{0} \bar{v}_{1}\right) \zeta^{2}+\cdots\right]\right. \\
& \left.\left.+\varepsilon^{7}\left(\bar{W}_{30}+\varepsilon \bar{W}_{31}+\cdots\right)\right\} E^{3}\right\}+O\left(\varepsilon^{10}\right)+\text { c.c. }, \\
& \quad \tilde{P}=\varepsilon^{7 / 2}\left\{\varepsilon^{3}\left(\bar{P}_{0}+\varepsilon P_{1}+\cdots\right) E+\varepsilon^{5 / 2}\left(\bar{P}_{20}+\varepsilon \bar{P}_{21}+\cdots\right) E^{2}\right. \\
& \left.\quad+\varepsilon^{27 / 2}\left(\bar{P}_{m 0}+\varepsilon \bar{P}_{m 1}+\cdots\right)+\varepsilon^{4}\left(\bar{P}_{30}+\varepsilon \bar{P}_{31}+\cdots\right) E^{3}\right\} \\
& \quad+O\left(\varepsilon^{8}\right)+\text { c.c. },
\end{aligned}
$$

where, now, quantities depend upon $r_{1}$ and $\zeta$. The amplitude equation we are seeking is obtained by substitution of (4.8)-(4.10) into the non-linear disturbance equations (2.3), equating coefficients of like powers of $\varepsilon$ from the resulting equations for the terms proportional to $E$, and finally solving for $\bar{W}_{1}$. The contribution coming from the terms of $O\left(E^{2}\right)$ and of $O\left(E^{3}\right)$ will be too small and thus neglected.

However, instead of substitution of (4.8)-(4.10), the $\bar{W}_{1}$ solution can also be determined in the following manner. The linear contribution to the $\bar{W}_{1}$ term will come in from $\left(U_{2}, V_{2}, W_{1}, P_{1}\right)$ from the fourth equations in (3.17) (3.20) after replacing $r$ by $\bar{r}$, as well as by expanding $W_{0}$ in equation (3.22) using (4.6) and extracting out $O(\varepsilon)$ terms. The non-linear contribution will be simply as in $W_{10}$ in equation (3.31), due to the argument given in [2]. Therefore, multiplying the last equations in (3.18) (and a similar one for $\tilde{V}$ ) by $i r \alpha_{0}$ and $i \beta_{0}$, respectively, adding the results and making use of the fourth equation in the continuity equation (3.17), we obtain the solution for $W_{1}$ after matching with the main deck as

$$
\begin{aligned}
& W_{1}=\gamma_{0}^{2} \Delta^{-3 / 4} D_{1} F_{1}\left(s_{1}\right)+i k_{1} \Delta^{-5 / 4}\left[r \alpha_{1} \bar{u}_{0}+\beta_{0} \bar{v}_{0}\right] s_{1} \\
& -i A_{1} \Delta^{-1 / 4}\left[r \alpha_{2} \bar{u}_{0}+\beta_{2} \bar{v}_{0}\right] s_{1}+A_{1} F_{4}\left(s_{1}\right)+A_{1} \omega_{0} F_{5}\left(s_{1}\right)+O\left(s_{1}^{2}\right) .
\end{aligned}
$$


$F_{4}\left(s_{1}\right)$ and $F_{5}\left(s_{1}\right)$ are obtained from equations of the form (3.23) with the right-hand sides in (3.23) replaced by $R H S 1$ and $R H S 2$, respectively. $R H S 1$ is in fact in the same form as that given in 2] for the stationary waves, and $R H S 2$ contains the contribution due to the non-stationary perturbations and is given by

$$
\begin{aligned}
& R H S 2=\Delta^{-3 / 4}\left\{\left(r \alpha_{1} \bar{u}_{0}+\beta_{1} \bar{v}_{0}\right)\left[F_{1}\left(s_{1}\right)-s_{1} F_{1}^{\prime}\left(s_{1}\right)\right]\right. \\
& -\Delta^{-1 / 2}\left(r \alpha_{0} \bar{u}_{2}+\beta_{0} \bar{v}_{2}\right) s_{1}^{2}\left[s_{1} F_{1}^{\prime}\left(s_{1}\right)-3 F_{1}\left(s_{1}\right)+3\right] \\
& \left.-i \Delta^{-1 / 2}\left[\gamma_{0} \beta_{0}^{2} F_{2}^{\prime}\left(s_{1}\right)+2 i \beta_{0} U^{0^{2}} \bar{u}_{0} \frac{F_{3}^{\prime}\left(s_{1}\right)}{U_{c}(0,0)}\right]-2 \frac{i k_{1}}{A_{1}} \Delta^{-1 / 4} s_{1}\right\} \\
& +\Delta^{-1 / 2} \omega_{0} F_{1}^{\prime}\left(s_{1}\right) .
\end{aligned}
$$

Next, expanding the $W_{0}$ solution in (3.22) using (4.6), and taking into consideration $A_{1}=\bar{A}_{1}\left(1-\varepsilon \frac{r_{1}}{\bar{r}}\right)$ with $\bar{A}_{1}$ obtained by replacing $r$ by $r_{1}$ in $A_{1}$, and eventually together with (4.7) adding the resulting $O(\varepsilon)$ terms into (4.11), the quantity $\bar{W}_{1}$ appearing in (4.9) is given by

$$
\begin{aligned}
& \bar{W}_{1}=\frac{i}{\bar{r}} \beta_{1} \bar{v}_{0} \Delta^{-1 / 4} r_{1} \bar{A}_{1} s_{1}+i k_{1} \Delta^{-5 / 4}\left[\bar{r} \alpha_{1} \bar{u}_{0}+\beta_{0} \bar{v}_{0}\right] s_{1} \\
& -i \beta_{2} \bar{v}_{0} \Delta^{-1 / 4} \bar{A}_{1} s_{1}-\bar{r} \bar{u}_{0} \Delta^{-1 / 4} s_{1} \frac{d \bar{A}_{1}}{d r_{1}}-\Delta^{3 / 4} \bar{A}_{2} \bar{A}_{1}^{*} s_{1} \\
& +\Delta^{-3 / 4} \bar{\gamma}_{0}^{2} F_{2}\left(s_{1}\right)\left[D_{1}-\frac{2}{\bar{r}} r_{1} C_{1}\right]-2 \frac{i}{\bar{r}} \beta_{0} U^{0^{2}} \bar{u}_{0} \frac{F_{3}\left(s_{1}\right)}{U_{c}(0,0)} \Delta^{-3 / 4} r_{1} \bar{A}_{1} \\
& -\frac{i}{\bar{r}} \omega_{0} F_{1}\left(s_{1}\right) r_{1} \bar{A}_{1}+F_{4}\left(s_{1}\right) \bar{A}_{1}+\omega_{0} F_{5}\left(s_{1}\right) \bar{A}_{1}+O\left(s_{1}^{2}\right) .
\end{aligned}
$$

Considering the last equation in the continuity equation (3.17), the zero-wall derivative constraint also holds for $\bar{W}_{1}$ and hence the subsequent relation arises:

$$
\begin{aligned}
& \frac{i}{\bar{r}} \beta_{1} \bar{v}_{0} \Delta^{-1 / 4} r_{1} C_{1}+i k_{1} \Delta^{-5 / 4}\left[\bar{r} \alpha_{1} \bar{u}_{0}+\beta_{0} \bar{v}_{0}\right] \frac{\beta_{0}^{2}}{\bar{\gamma}_{0}}-i \beta_{2} \bar{v}_{0} \Delta^{-1 / 4} C_{1} \\
& -\bar{r} \bar{u}_{0} \Delta^{-1 / 4} \frac{d C_{1}}{d r_{1}}-\Delta^{3 / 4} C_{2} C_{1}^{*} \frac{\bar{\gamma}_{0}}{\beta_{0}^{2}}+\Delta^{-3 / 4} \beta_{0}^{2} \bar{\gamma}_{0} I_{2}\left[D_{1}-\frac{2}{\bar{r}} r_{1} C_{1}\right] \\
& -\frac{i}{\bar{r}} \beta_{0} U^{0^{2}} \bar{u}_{0} I_{3} \Delta^{-3 / 4} r_{1} C_{1}-\frac{i}{\bar{r}} \omega_{0} I_{1} r_{1} C_{1}+\left\{I_{4}+\omega_{0} I_{5}\right\} C_{1}=0,
\end{aligned}
$$

for which $I_{4}$ and $I_{5}$ are given by a complex contour integration as

$$
\begin{aligned}
& I_{4}=F_{4}^{\prime}(0)=\frac{1}{2 U_{c}(0,0)} \int_{0}^{\infty} \theta U_{c}(0, \theta) R H S 1\left(\sqrt{2} s_{1}=\theta\right) d \theta, \\
& I_{5}=F_{5}^{\prime}(0)=\frac{1}{2 U_{c}(0,0)} \int_{0}^{\infty} \theta U_{c}(0, \theta) R H S 2\left(\sqrt{2} s_{1}=\theta\right) d \theta .
\end{aligned}
$$

It should be remarked here that, by a direct matching of the solution $W_{0}$ in (3.22) with that of the main-deck solution in (3.8), the constant $k_{1}$ is found to be $k_{1}=$ $-i\left[r \alpha_{0} \bar{u}_{1}+\beta_{0} \bar{v}_{1}\right] \bar{B}_{1}$, with $\bar{B}_{1}=\frac{\bar{\gamma}_{0} D_{1}}{\beta_{0}^{2}}$, and thus the $k_{1}$ and $D_{1}$ terms appearing in (4.14) are taken care of together with the consideration of the eigenrelation (3.25).

We can now write (4.14) in the form

$$
\frac{d C_{1}}{d r_{1}}=(a+i b) r_{1} C_{1}+(c+i d) C_{1}\left|C_{1}\right|^{2}+(e+i f) C_{1},
$$

where with the help of (3.25) the constants $a, b, c, d, e$ and $f$ in (4.16) are given by

$$
\begin{aligned}
& a=-\frac{\bar{\gamma}_{0} \beta_{0}^{2} I_{2}}{\sqrt{2} \bar{r}^{2} \bar{u}_{0} \Delta_{0}^{1 / 2}}, \quad b=\frac{\beta_{1} \bar{v}_{0}}{\bar{r}^{2} \bar{u}_{0}}-a-\frac{\beta_{0} \Phi_{0}}{\bar{r}}, \\
& c=2 \frac{\bar{\gamma}_{0}^{2} \Delta_{0}^{5 / 2}}{\bar{r} \bar{u}_{0} \beta_{0}^{4}} \frac{\left(\kappa_{1}-\kappa_{2}\right)}{\left(\kappa_{1}^{2}+\kappa_{2}^{2}\right)}, \quad d=-2 \frac{\bar{\gamma}_{0}^{2} \Delta_{0}^{5 / 2}}{\bar{r} \bar{u}_{0} \beta_{0}^{4}} \frac{\left(\kappa_{1}+\kappa_{2}\right)}{\left(\kappa_{1}^{2}+\kappa_{2}^{2}\right)}, \\
& e+i f=\frac{1}{\bar{r} \bar{u}_{0} \Delta^{-1 / 4}}\left(-i \beta_{2} \bar{v}_{0} \Delta^{-1 / 4}+I_{4}+\omega_{0} I_{5}\right),
\end{aligned}
$$

and $\Delta_{0}=i \Delta, \kappa_{1}=(4-\sqrt{2}) \bar{\gamma}_{0} \beta_{0}^{2} I_{2}+\left(2^{3 / 4}-\sqrt{2}\right) \cos (\pi / 8) \omega_{0} I_{1} \Delta_{0}^{3 / 4}$ and $\kappa_{2}=$ $(1-\sqrt{2}) \beta_{0} \bar{u}_{0} U^{0^{2}} I_{3}+\left(2^{3 / 4}-\sqrt{2}\right) \sin (\pi / 8) \omega_{0} I_{1} \Delta_{0}^{3 / 4}$. The variation of parameter $c$ in equation (4.17) is demonstrated graphically versus the scaled parameter $\gamma$ in Figure 6 . 


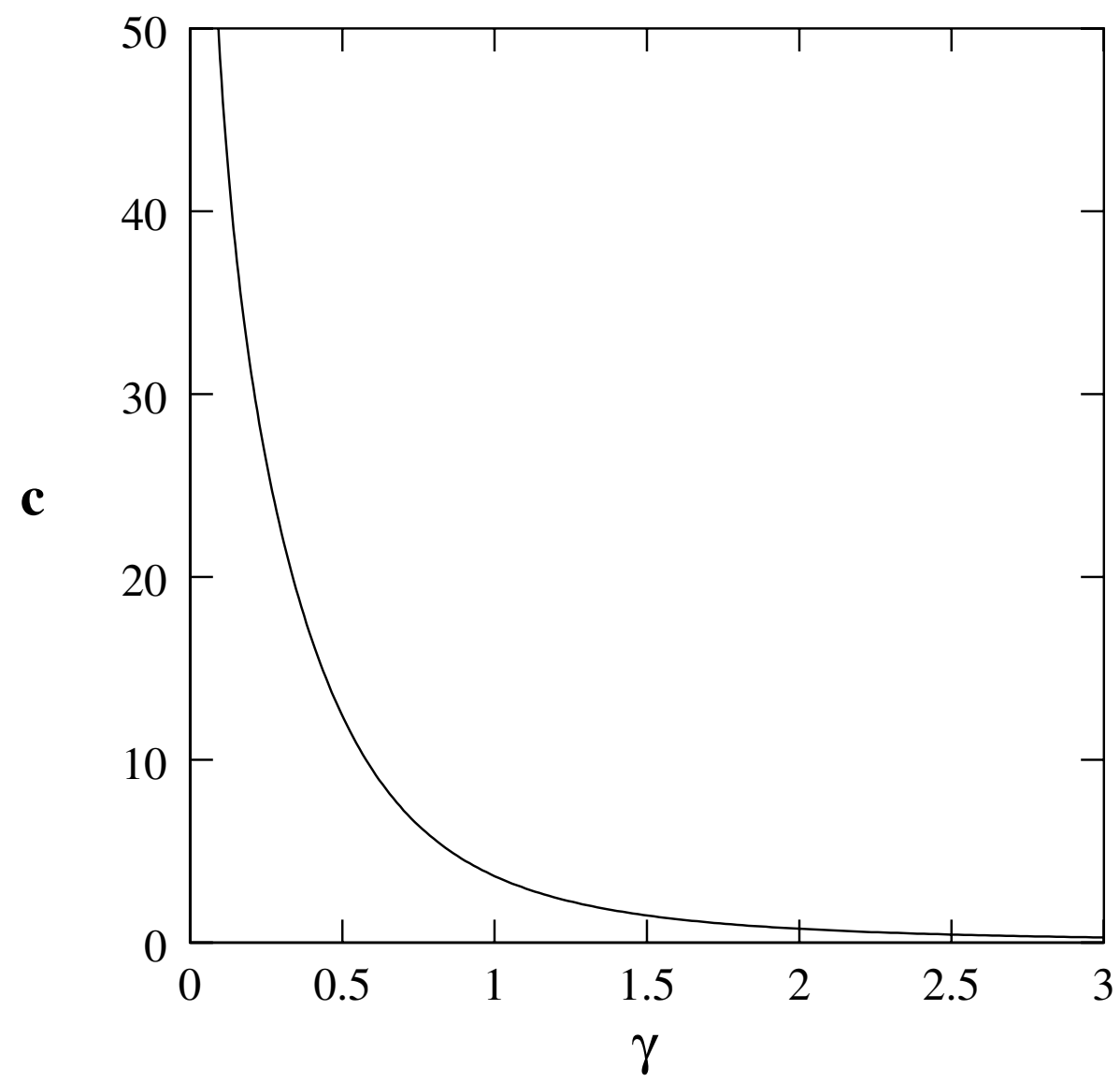

FIG. 6. The variation of parameter $c$ in equation (4.17) is demonstrated versus the scaled parameter $\gamma$.

Afterwards, multiplying (4.16) through by $C_{1}^{*}$ and adding the complex conjugate together with the substitution $r_{2}=r_{1}+\frac{e}{a}$ gives

$$
\frac{d\left|C_{1}\right|^{2}}{d r_{2}}=2 a r_{2}\left|C_{1}\right|^{2}+2 c\left|C_{1}\right|^{4} \text {. }
$$

Since equation (4.18) is of Bernoulli type, the form of the solution depends on the signs of $a$ and $c$, which are in turn related to $\bar{\gamma}_{0}$ and $\omega_{0}$. However, equation (4.17) shows that regardless of the sign of the frequency term, $a$ is always negative and hence the amplitude of the solution, $\left|C_{1}\right|$, increases or decreases (see (4.18)) depending on whether $r$ is less than or greater than the neutral value, which will also involve the sign of $e$. With the change of variables $\left|C_{1}\right|^{2}=\frac{\sqrt{-a}}{2 c} y$ and $r_{2}=\frac{x}{\sqrt{-a}}$, (4.18) reduces to

$$
\frac{d y}{d x}=-2 x y+y^{2},
$$

whose solution can be formulated as

$$
y(x)=\frac{2 y_{0} e^{-x^{2}}}{2-y_{0} \sqrt{\pi} \operatorname{erf} x},
$$


where $y_{0}=y(0), x=0$ corresponds to the neutral position and erf $x=\frac{2}{\sqrt{\pi}} \int_{0}^{x} e^{-t^{2}} d t$. As a result, the amplitude function is given by

$$
\left|C_{1}\right|=\sqrt{\frac{y_{0} \sqrt{-a}}{c}} \frac{e^{-x^{2} / 2}}{\left(2-y_{0} \sqrt{\pi} \operatorname{erf} x\right)^{1 / 2}},
$$

where $x=\sqrt{-a} r_{2}$ and $y_{0}=\left.\frac{2 c}{\sqrt{-a}}\left|C_{1}\right|^{2}\right|_{x=0}$.

Equation (4.19) together with (4.21) reveals that the amplification or decay of the amplitude takes place around a critical point $x_{d}$, where $x_{d}$ is given by

$$
y_{0} \frac{e^{-x_{d}^{2}}}{2-y_{0} \sqrt{\pi} \operatorname{erf} x_{d}}=x_{d} .
$$

It is also seen from equation (4.17) and Figure 6 that $c$ is always positive for all the frequencies, and thus regarding also equation (4.18), it is clear that the non-linearity has a destabilizing impact for both positive and negative frequency waves, while more destabilizing for positive frequencies.

With a similar argument as in [2], the amplitude function (4.21) becomes finite for all $x$ as long as $y_{0}<2 / \sqrt{\pi}$. On the other hand, $\left|C_{1}\right|$ will be unbounded for $y_{0} \geq 2 / \sqrt{\pi}$, and there happens to be a cut-off at the value of $x_{e}$, where $x_{e}$ is given by

$$
y_{0}=2 /\left(\sqrt{\pi} \operatorname{erf} x_{e}\right)
$$

Therefore, $y_{0}=2 / \sqrt{\pi}$ represents a threshold between the solutions always growing and those decaying to zero. In terms of the amplitude function (4.21), this threshold depends upon the frequency and is given by

$$
\mid C_{1} \|_{x=0}=\left(\frac{-a}{\pi c^{2}}\right)^{1 / 4}
$$

From this finding, and also making use of (4.17), for positive frequencies with the scaled wavenumber $\gamma$ small ( $\Omega \sim \gamma^{1 / 4}$ from equation (3.27), see also Figure 2), the initial amplitude $\mid C_{1} \|_{x=0}$ will be order of magnitude of $\gamma^{7 / 8}$, whereas for negative frequencies with $\gamma$ large $\left(\Omega \sim-\gamma^{9 / 4}\right.$ from equation (3.27), see also Figure 2), it will be order of magnitude of $\gamma^{15 / 8}$. This order of magnitude analysis implies that for the total amplification of the perturbations received into the three-dimensional rotating-disk boundary layer a smaller amplitude will be sufficient for the positive frequency waves but not for the negative ones. Thus, the destabilizing influences of non-linearity are greater for a positive frequency disturbance than for a negative one, resulting in the fact that for the negative frequencies close to the neutral location the non-linear effects are less important. This may also explain why the travelling modes dominated in the early stages of instability in the experiment of [32] for the natural transition process over a smooth rotating disk. If the transition is forced with a roughness element on the disk, then the non-stationary disturbances having finite amplitude were found to cause the transition in 33. The above outcome thus enables us to conclude that turbulence will first take place through the positive frequency waves, if of course the lower branch modes calculated here dominate over the inviscid upper branch modes of [12. If this is the case, then the non-stationary short-wavelength unstable modes described in this paper could be observed experimentally as in [19] and [32, 33. However, it is an inevitable fact that at sufficiently large 
Reynolds numbers there will always be a broad spectrum of unstable inviscid modes with order-one growth rates which dominate over the transition process completely.

5. Conclusions. In the present study a rational asymptotic expansion procedure which, as opposed to the conventional parallel-flow approximation, rigorously takes care of the non-linearity, non-parallelism and influences of viscous and Coriolis forces, has been pursued successfully to investigate the behavior of the non-stationary short-wavelength small frequency lower branch modes of the disturbances imposed on the incompressible three-dimensional boundary layer flow due to a rotating disk. First of all, the effects of non-parallelism have been determined on the evolution of linear lower branch modes. Secondly, the influences of the non-linearity have been searched on the time-dependent solutions, but quite close to the stationary neutral waves having vanishingly small shear stress on the wall. On this level, moving from the neutral location, the amplitude function of a growing or decaying perturbation has been determined as a function of the distance from the position of neutral stability. The most important conclusion from the present study is that, regardless of the positive or negative frequency waves, the non-linearity acts in favor of destabilization of the three-dimensional rotating-disk boundary layer flow. The destabilizing influence of the non-linearity is particularly pronounced for the positive short-wavelength frequency waves.

Comparisons show that, although the parallel-flow approximation to the full linearized equations is only valid for $R e \rightarrow \infty$, nevertheless, to the order calculated the numerical and asymptotic results are graphically consistent. This consistency strongly suggests that the structure of the expansions for different quantities in each asymptotic regime of the triple deck is also appropriate to more general three-dimensional boundary layers (such as the Ekman and Bödewat layers), requiring only minor modifications in the flows of practical interest. However, it should be stressed here that the structure of the lower branch modes described here is formed both with the fact of having a vanishing shear at the wall in the effective mean cross-flow profile, and by a balance between the viscous and Coriolis forces. Therefore, unlike the upper branch modes, lower branch modes of the kind found here may not even exist in other three-dimensional boundary layers possessing no Coriolis term. This is as a result of the fact that the lower branch modes are governed by a relation determined from parabolic cylinder functions, rather than Airy functions or some other functions involved in eigenrelations in three-dimensional classical boundary layers; see for instance 38 .

The solution of the eigenrelation governing the effective wavenumber and frequency has not only demonstrated clearly the appearance of a double mode for some certain positive frequency waves, but also proved the existence of a critical frequency above which no neutral modes can occur. Non-parallel effects are overall found to be destabilizing. Moreover, in rotating-disk flow, the non-linear influences are more important for the lower branch modes close to the neutral locations than for the inviscid upper branch modes (though the critical layers in the upper branch might yield a significant non-linearity at low amplitudes).

In line with the stationary mode of [2], a threshold amplitude of the disturbances has been found to exist at the position of neutral stability of the lower branch modes. If 
at the position of neutral stability, the amplitude of any disturbance is larger than this specific value, the solutions are most likely to grow in size driving the flow into turbulence, as the distance from the neutral stability location is increased. Equation (4.21) proves that the initial amplitude of the modes leading to an infinite growth is much smaller for the positive frequency waves as compared to the negative ones. Thus, in compliance with the experiments of [32, 33, non-stationary waves having positive frequencies with sufficiently small amplitude near the neutral location are of significance in applications. These unstable modes are most probably the ones observed also in the experiment of [19. If, however, the amplitude of a disturbance is smaller than the threshold amplitude, it decays eventually to zero after an initial growth as the distance from the position of neutral stability is increased, leaving the flow stable. In this case, of course the unstable inviscid mode observed to be dominant in the experiment of [12] will definitely be in play.

Another point which deserves mentioning is that as the difference in behavior between positive and negative frequencies is discovered here, some differences can be expected between waves which rotate faster or slower than the disk, an issue that requires further work.

It might also be interesting to explore whether the lower branch modes calculated analytically here can give rise to absolute growth of the disturbances, as in the works of [9, 39]. This certainly requires the multiple layer analysis of [9] and [40] (see also [41]), which seems most appropriate for the neutral waves found in this work. Finally, it was shown in 42 that the non-linearity is also destabilizing in the compressible rotating-disk boundary layer flow as far as the stationary modes are concerned. The effects of wall insulation and isothermal wall were also found to be destabilizing, indicating the greatest likelihood of instability through highly cooled walls. How the compressibility will alter the character of the time-dependent modes accounted for in this study warrants further work and is currently being investigated.

\section{REFERENCES}

[1] P. Hall, An asymptotic investigation of the stationary modes of instability of the boundary layer on a rotating-disk, Proc. Roy. Soc. London Ser. A, 406 (1986), 93-106.

[2] S. O. MacKerrel, A nonlinear asymptotic investigation of the stationary modes of instability of the three-dimensional boundary layer on a rotating disk, Proc. Roy. Soc. London Ser. A, 413 (1987), 497-513. MR0915128(89a:76038)

[3] R. J. Lingwood, Absolute instability of the boundary layer on a rotating disk, J. Fluid Mech., 299, (1995), 17-33. MR.1351381 (96e:76061)

[4] R. J. Lingwood, An experimental study of absolute instability of the rotating-disk boundary layer flow, J. Fluid Mech., 314, (1996), 373-405.

[5] R. J. Lingwood, On the application of the Briggs' and steepest-descent methods to a boundary layer flow, Stud. Appl. Math., 98, (1997), 213-254. MR1441304 (97k:76034)

[6] M. Turkyilmazoglu, Linear absolute and convective instabilities of some two- and three-dimensional flows, Ph.D. Thesis, (1998), University of Manchester.

[7] M. Turkyilmazoglu, J. W. Cole and J. S. B. Gajjar, Absolute and convective instabilities in the compressible boundary layer on a rotating disk, Theoret. Comput. Fluid Dyn., 14, (2000), 21-37.

[8] M. Turkyilmazoglu and J. S. B. Gajjar, Direct spatial resonance in the laminar boundary layer due to a rotating-disk, SADHANA-ACAD P ENG S., 25, (2000), 601-617. 
[9] M. Turkyilmazoglu and J. S. B. Gajjar, An analytic approach for calculating absolutely unstable inviscid modes of the boundary layer on a rotating disk, Stud. Appl. Math., 106, (2001), 419-435. MR 1825844 (2002a:76168)

[10] B. Pier, Finite-amplitude crossflow vortices, secondary instability and transition in the rotating-disk boundary layer, J. Fluid Mech., 487, (2003), 315-343. MR2017795(2004i:76186)

[11] A. J. Cooper and P. W. Carpenter, The stability of rotating-disk boundary layer flow over a compliant wall. Part II. Absolute instability , J. Fluid Mech., 350, (1997), 261-270. MR1481902 (98k:76056)

[12] N. Gregory, J. T. Stuart and W. S. Walker, On the stability of three-dimensional boundary layers with applications to the flow due to a rotating disk, Philos. Trans. Roy. Soc. London Ser. A, 248, (1955), 155-199. MR0072616 (17:311c)

[13] M. R. Malik, S. P. Wilkinson and S. A. Orszag, Instability and transition in rotating-disk flow, AIAA Journal, 19, (1981), 1131-1138.

[14] M. R. Malik, D. I. A. Poll, Effect of curvature on three-dimensional boundary-layer stability, AIAA Journal, 23, (1985), 1362-1369. MR0798889 (87a:76058)

[15] M. R. Malik, The neutral curve for stationary disturbances in rotating-disk flow, J. Fluid Mech., 164, (1986), 257-287. MR0844675 (87f:76113)

[16] S. P. Wilkinson and M. R. Malik, Stability experiments in rotating-disk flow, AIAA Pap., 1760, (1983).

[17] S. P. Wilkinson and M. R. Malik, Stability experiments in the flow over a rotating-disk, AIAA Journal, 23, (1985), 588-595.

[18] A. J. Faller, Instability and transition of disturbed flow over a rotating-disk, J. Fluid Mech., 230, (1991), 245-269.

[19] B. I. Federov, G. Z. Plavnik, I. V. Prokhorov and L. G. Zhukhovitskii, Transitional flow conditions on a rotating-disk, J. Eng. Phys., 31, (1976), 1448-1453.

[20] P. Balakumar and M. R. Malik, Travelling disturbances in rotating-disk flow, Theoret. Comput. Fluid Dyn., 2, (1990), 125-137.

[21] K. Stewartson, On the flow near the trailing-edge of a flat plate, MATHEMATICA, 16, (1969), $106-121$.

[22] K. Stewartson and P. G. Williams, Self-induced separation, Proc. Roy. Soc. London Ser. A, 312, (1969), 181-206

[23] F. T. Smith, On the high Reynolds number theory of laminar flows, IMA Journal of Applied Mathematics, 28 (1982), 207-281. MR0666155 (83g:76047)

[24] Vladimir V. Sychev, A. I. Ruban, Victor V. Sychev and G. L. Korolev, Asymptotic theory of separated flows, Cambridge University Press, (1998). MR1659235(2000d:76049)

[25] C. C. Lin, On the stability of two-dimensional parallel flows. Part III. Stability in a viscous fluid, Quart. J. Mech. Appl. Math., 3, (1946), 277-301. MR0014894 (7:346b)

[26] F. T. Smith, On the non-parallel flow stability of the Blasius boundary layer, Proc. Roy. Soc. London Ser. A, 366, (1979), 91-109.

[27] F. T. Smith and R. J. Bodonyi, Nonlinear critical layers and their development in streaming-flow stability, J. Fluid Mech., 118, (1982), 165-185. MR0663988 (83g:76055)

[28] A. P. Bassom and J. S. B. Gajjar, Nonstationary cross-flow vortices in three-dimensional boundarylayer flows, Proc. Roy. Soc. London Ser. A, 417, (1988), 179-212. MR0944282 (89e:76036)

[29] M. Turkyilmazoglu and J. S. B. Gajjar, Upper branch nonstationary modes of the boundary layer due to a rotating disk, Applied Mathematics Letters, 14, (2001), 685-690. MR1836070 (2002d:76050)

[30] Y. Kohama, Crossflow instability in rotating-disk boundary layer, AIAA Pap., 1340, (1987).

[31] Y. Kohama, Crossflow instability in a spinning disk boundary layer, AIAA Journal, 31, (1992), 212-214.

[32] S. L. G. Jarre and M. P. Chauve, Experimental study of rotating-disk instability. I. Natural flow, Phys. Fluids., 8, (1996), 496-508.

[33] S. L. G. Jarre and M. P. Chauve, Experimental study of rotating-disk instability. II. Forced flow, Phys. Fluids., 8, (1996), 2985-2994.

[34] J. J. Healey, A new convective instability of the rotating-disk boundary layer with growth normal to the disk, J. Fluid Mech., 560, (2006), 279-310.

[35] M. Abramowitz and I. A. Stegun, Handbook of mathematical functions, Dover Publications, (1955).

[36] A. I. Ruban, Nonlinear equation for the amplitude of the Tollmien-Schlichting wave in the boundary layer, Fluid Dyn. Res., 19, (1983), 709-716. 
[37] P. Hall and F. T. Smith, On the effects of nonparallelism, 3-dimensionality, and mode interaction in nonlinear boundary-layer stability, Stud. Appl. Math., 71, (1984), 91-120.

[38] S. N. Timoshin, On short-wavelength instabilities in three-dimensional classical boundary layers, Eur. J. Mech., B/Fluids, 14, (1995), 409-437. MR1349727 (96e:76064)

[39] M. Turkyilmazoglu, Asymptotic calculation of inviscidly absolutely unstable modes of the compressible boundary layer on a rotating disk, Applied Mathematics Letters, 19, (2006), 795-800. MR.2232257

[40] J. S. B. Gajjar, Nonlinear evolution of a 1st mode oblique wave in a compressible boundary layer. Part 1. Heated/cooled walls, IMA Journal of Applied Mathematics, 53, (1994), 221-248. MR 1314256 (95i:76089)

[41] M. Choudhari, Long-wavelength asymptotics of stationary crossflow instability in incompressible and compressible boundary layer flows, AIAA Pap., 2137, (1996).

[42] S. O. Seddougui, A nonlinear investigation of the stationary modes of instability of the threedimensional compressible boundary layer due to a rotating disk, Quart. J. Mech. Appl. Math., 43, (1990), 467-497. MR1081299 (91j:76055) 